\title{
Convergence of Vestibular and Neck Proprioceptive Sensory Signals in the Cerebellar Interpositus
}

\author{
Hongge Luan, ${ }^{1,3}$ Martha Johnson Gdowski, ${ }^{1}$ Shawn D. Newlands, ${ }^{1,3}$ and Greg T. Gdowski ${ }^{1,2}$ \\ Departments of ${ }^{1}$ Neurobiology and Anatomy, ${ }^{2}$ Biomedical Engineering, and ${ }^{3}$ Otolaryngology, University of Rochester, Rochester, New York 14642
}

The cerebellar interpositus nucleus (IN) contributes to controlling voluntary limb movements. We hypothesized that the vestibular signals within the IN might be transformed into coordinates describing the body's movement, appropriate for controlling limb movement. We tested this hypothesis by recording from IN neurons in alert squirrel monkeys during vestibular and proprioceptive stimulation produced during (1) yaw head-on-trunk rotation about the $\mathrm{C} 1-\mathrm{C} 2$ axis while in an orthograde posture and (2) lateral side-to-side flexion about the $\mathrm{C} 6-\mathrm{T} 3$ axis while in a pronograde posture. Neurons (44/67) were sensitive to vestibular stimulation (23/44 to rotation and translation, 14/44 to rotation only, 7/44 to translation only). Most neurons responded during contralateral movement. Neurons (29/44) had proprioceptive responses; the majority (21/29) were activated during neck rotation and lateral flexion. In all 29 neurons with convergent vestibular and neck proprioceptive input those inputs functionally canceled each other during all combined sensory stimulation, whether in the orthograde or pronograde posture. These results suggest that two distinct populations of IN neurons exist, each of which has vestibular sensitivity. One population carries vestibular signals that describe the head's movement in space as is traditional for vestibular signals without proprioceptive signals. A second population of neurons demonstrated precise matching of vestibular and proprioceptive signals, even for complicated stimuli, which activated the semicircular canals and otolith organs and involved both rotation and flexion in the spine. Such neurons code body (not head) motion in space, which may be the appropriate platform for controlling limb movements.

\section{Introduction}

Vestibular and proprioceptive sensory signals are used in the control of postural reflexes and voluntary movements (Magnus, 1924). Such signals may be important in estimating the body's motion in space to produce accurate volitional movements (e.g., consider a runner who produces an arm movement to acquire a glass of water from a race attendant). However, the construction of an estimate of the body's movement in space from sensory signals is not straightforward. Primary vestibular afferent signals are inadequate because they encode movements of the head in space. Head movements with respect to the trunk, whether voluntary (e.g., turning the head toward an attendant) or reflexive, produce vestibular afferent signals. Motion of the head and body in space (e.g., running) also stimulates vestibular afferents. Fundamental questions include which of these vestibular signals are important in controlling limb movements and how does the CNS computationally construct a signal representing body movement

\footnotetext{
Received July 19, 2012; revised Nov. 17, 2012; accepted Nov. 25, 2012.

Author contributions: H.L. designed research; H.L. performed research; H.L. analyzed data; M.J.G., S.D.N., and G.T.G. wrote the paper.

This work was supported by the National Institute on Deafness and Other Communication Disorders (R01DC006498 and P30-DC05409) and the National Eye Institute (R01EY008041). We thank Robert McCrea for his support during development of the experiments and in data acquisition. We also thank Tim Belton and Vladimir Marlinski for their assistance in all aspects of the experiments. These experiments were performed at the University of Chicago in the Department of Neurobiology.

Correspondence should be addressed to Dr. Shawn D. Newlands, University of Rochester, Department of Otolaryngology, 601 Elmwood Avenue, Box 629, Rochester, NY 14642. E-mail:Shawn_Newlands@urmc.rochester.edu.

DOI:10.1523/JNEUROSCI.3460-12.2013

Copyright $\odot 2013$ the authors $\quad 0270-6474 / 13 / 331198-13 \$ 15.00 / 0$
}

in space from the convergence of multiple types of vestibular afferent sensory signals?

Many cerebellar regions use visual, somatosensory, and vestibular sensory signals to control movements of the body (Eccles et al., 1974; Ito, 1984). Recent studies of the vermis and rostral fastigial nucleus ( $\mathrm{rFN}$ ) have shown that vestibular sensory signals in these regions are transformed into signals that describe the body's movement in space (Shaikh et al., 2005a,b; Brooks and Cullen, 2009). If these transformations are important in the execution of reflexive movements involving these medial cerebellar structures, one might also expect such transformations in lateral cerebellar regions involved in controlling voluntary limb movements, such as the cerebellar dentate and interpositus nuclei (INs). The IN specifically targets limb movement premotor regions, such as the red nucleus, that use sensory signals to regulate movement (Houk et al., 1993; Miller et al., 1993). The integration of vestibular and neck proprioceptive signals within these regions has been described in decerebrate cats (Boyle and Pompeiano, 1979, 1980b; Chan et al., 1982). However, the nature of this sensory integration in awake nonhuman primates remains unknown.

In this study, we have quantified and compared the vestibular and neck proprioceptive signals in the IN that would be generated during yaw head-on-trunk movement. While many IN neurons with vestibular sensitivity did not receive proprioceptive input, most received neck signals that combined antagonistically when the head was rotated about the body. The results of this study suggest that IN neurons carry neck proprioceptive and vestibular sensory signals that cancel each other during head-on-trunk 
movements. These findings suggest that reference frame transformations in the IN may occur similarly to transformations seen in the rFN (Brooks and Cullen, 2009). These findings also demonstrate that single neurons in the cerebellum can code for body movements even during complicated 3D head and body movements that involve multiple vestibular end organs and complex neck movements across several cervical levels. These findings thus expand the possibilities of how the IN might contribute to control of limb movements during complicated tasks that require coordination of the head, body and limbs.

\section{Materials and Methods}

Many of the methods have been described previously (Gdowski and McCrea, 1999, 2000; Belton and McCrea, 2000). All protocols were approved by the Institutional Animal Care and Use Committees at the University of Chicago and the University of Rochester. The animals were housed under conditions compliant with the standards of the National Institutes of Health (NIH publication No. 86-23) as stated in the Guide for the Care and Use of Laboratory Animals (2003) and the Association for Assessment and Accreditation of Laboratory Animal Care International.

\section{Surgical preparation}

Three female adult squirrel monkeys were surgically prepared for chronic recordings of head and eye movements, single-unit recording, and bilateral stimulation of the vestibular labyrinth. Under inhalation anesthesia (isoflurane), a stainless steel plate was attached to the cranium for controlling the head's position and for holding the connectors of eye coils and stimulating electrodes. A small portion of the parietal bone was removed within a recording chamber $\left(\sim 100 \mathrm{~mm}^{2}\right)$ to permit electrode insertion. In a second surgery, fine wire coils were implanted in one eye for recording gaze position using the magnetic search coil technique (Robinson, 1964). A bipolar stimulating electrode was implanted on both promontory bones adjacent to the round window to enable activation of both vestibular nerves. The ground of each bipolar stimulating electrode consists of a small round ball on the end that was placed on the floor of the middle ear cavity.

In two animals, two sets of four stimulating electrodes were bilaterally implanted using stereotaxic coordinates into the following thalamic nuclei: ventralis lateralis (VL), lateralis dorsalis (LD), and medialis dorsalis (MD). Details of the implantation procedure were described previously (Marlinski and McCrea, 2008a,b). The four equally spaced electrodes $(1.5 \mathrm{~mm})$ were placed along the coronal plane (anteroposterior $(\mathrm{AP}),=$ $6.0 \mathrm{~mm}$ ). The most medial electrode (mediolateral $(\mathrm{ML})=1.0 \mathrm{~mm}$, dorsoventral $(\mathrm{DV})=7.4 \mathrm{~mm}$ ) was located in the MD nucleus. The second electrode (DV $=8.4 \mathrm{~mm}$ ) was located in the LD nucleus. The remaining two electrodes were located at a common depth $(\mathrm{DV}=6.4$ $\mathrm{mm}$ ) in the VL nucleus. Electrical stimulation was performed with the lateral most electrode in VL serving as the negative lead and the other three electrodes serving as the positive leads. Thus, the current delivered during stimulation was always directed away from the midline toward the ipsilateral VL nucleus. The coordinates for stereotaxic implantation were based on prior recordings in the vestibular nucleus (VN), VL, and the nucleus ventroposterior lateralis.

\section{Experimental setup}

Recordings were conducted in a light-proof, sound-attenuated room equipped with a linear sled (Trilogy Systems) that was mounted on top of a motor that was used to produce yaw rotation about an earth-vertical axis (Kollmorgan). All experiments in this study were performed in the dark to exclude influences of visual input.

The experiments were designed to characterize the convergence of vestibular and proprioceptive signals that are produced during head-ontrunk movements that incorporate lateral flexion of the vertebral column. This was accomplished by developing a system in which the animal's posture could be transitioned from an orthograde to a pronograde posture while recording single neurons in the cerebellar interpositus. All of the animals wore a custom-fitted vest and stood on a perch that was mounted on top of a platform on the experimental apparatus. Each vest was designed to experimentally control trunk movements produced around the shoulder girdle and/or the pelvic girdle. The perch itself consisted of two semicircular handles: one grasped by the hands and the other by the feet. The portion of the vest in the region of the pelvic girdle was attached to the superstructure at the center of the experimental apparatus to minimize movements of the lower torso. The headmounted plate was coupled to a series of rods to control the rotational axis of the head and upper torso with respect to the pelvic girdle. The first rod (vertical) defined an earth-vertical rotational axis with respect to the spinal column. Rotations about this axis produced yaw rotation in space. A second rod (horizontal) defined the distance (Fig. 1A, $r$ ) between the head and the rotational axis of the system.

At the beginning of each session, the body was placed in an orthograde posture so that the rotational axis of the vertical rod was aligned with the $\mathrm{C} 1-\mathrm{C} 2$ axis and the center of the earth-vertical rotational axis of the turntable. All of the paradigms performed while in this posture are referred to as on-axis paradigms as described later in this section, because the axis of rotation is through the $\mathrm{C} 1-\mathrm{C} 2$ axis (Fig. $1 B$ ). After the on-axis paradigms were completed, the subject was gently transitioned into a pronograde posture. This experimental manipulation was accomplished by using the horizontal rod to translate the subject's head anteriorly, thereby increasing the distance between the center of the horizontal rotational axis of the turntable and center of the head. This forward translation of the head displaced the vertebral level at which rotation occurred from the $\mathrm{C} 1-\mathrm{C} 2$ axis to more inferior vertebral levels in close proximity to C6. All of the paradigms performed while in this posture are referred to as eccentric off-axis because the center of rotation is $8 \mathrm{~cm}$ behind the center of the $\mathrm{C} 1-\mathrm{C} 2$ axis. Natural head movement produced while in this posture can incorporate both lateral flexion about $\mathrm{C} 6-\mathrm{T} 3$ and rotation about the $\mathrm{C} 1-\mathrm{C} 2$ axis. The horizontal bar was used to displace the head a specific distance from the horizontal rotational axis. Its use limited movements to those produced by lateral flexion and prevented $\mathrm{C} 1-\mathrm{C} 2$ rotational motion. Head movements produced while in this posture resulted in combined rotation and translation of the vestibular end organs (located approximately along the interaural plane) in space. Subject's heads were positioned $8 \mathrm{~cm}$ in front of the earth-vertical rotational axis to elicit uniform activation of otolith and canal end organs during yaw rotations across all subjects. This $8 \mathrm{~cm}$ displacement yielded an earthvertical rotational axis that was always located between $\mathrm{C} 6$ and T3, but the precise location varied slightly between subjects due to differences in their total vertebral length.

\section{Recording of eye and head movement}

Gaze and head position were measured throughout all experiments. Gaze position was measured with the implanted scleral search coil. Head position was measured with a second coil placed on the vertical rod that defined the earth-vertical rotational axis of the system. The gaze coil was located $\sim 2 \mathrm{~cm}$ anterior to the center of the fields during on-axis paradigms and $10 \mathrm{~cm}$ anterior to the center of the fields during eccentric off-axis paradigms. The system was calibrated while the animal was located in both positions. Only small differences in gaze coil sensitivity were observed between the two positions $(<10 \%)$. Differences in sensitivity were scaled accordingly while in each position. The sensitivity of the head position coil was not dependent upon the animal's position because it was located on the vertical rod and was always located in the center of the coil system. Horizontal eye position was calculated off-line as the algebraic difference between horizontal gaze and horizontal head position. Nonetheless, it is important to note that all neurons reported here were not sensitive to eye movements.

\section{Data acquisition}

A data acquisition system (Cambridge Electronics, 1401) was used in conjunction with customized acquisition software (Spike2). Analog signals including horizontal and vertical eye position, head and trunk position, turntable velocity, linear translation position, and acceleration were lowpass filtered $(500 \mathrm{~Hz})$ and digitized with analog to digital converters of the acquisition system (sampling rate: $1 \mathrm{kHz}$ ). Digital to analog converters of the acquisition system were used to produce stimulus wave- 
A
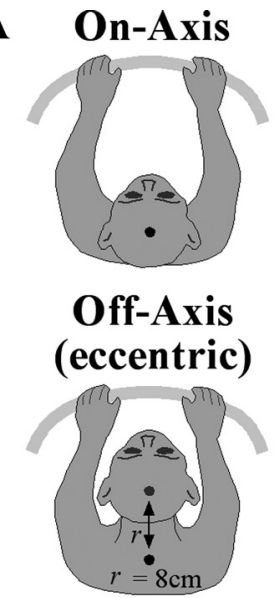

C Off-Axis

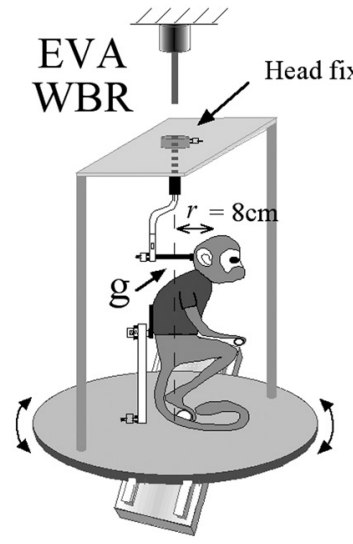

B On-Axis

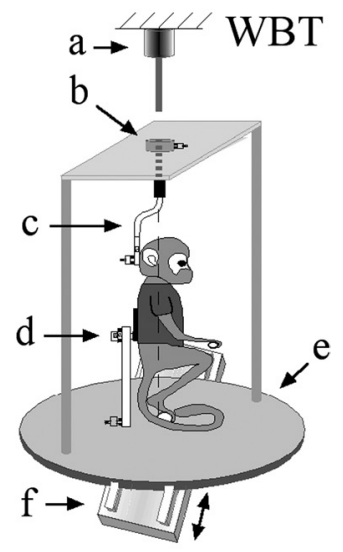

On-Axis
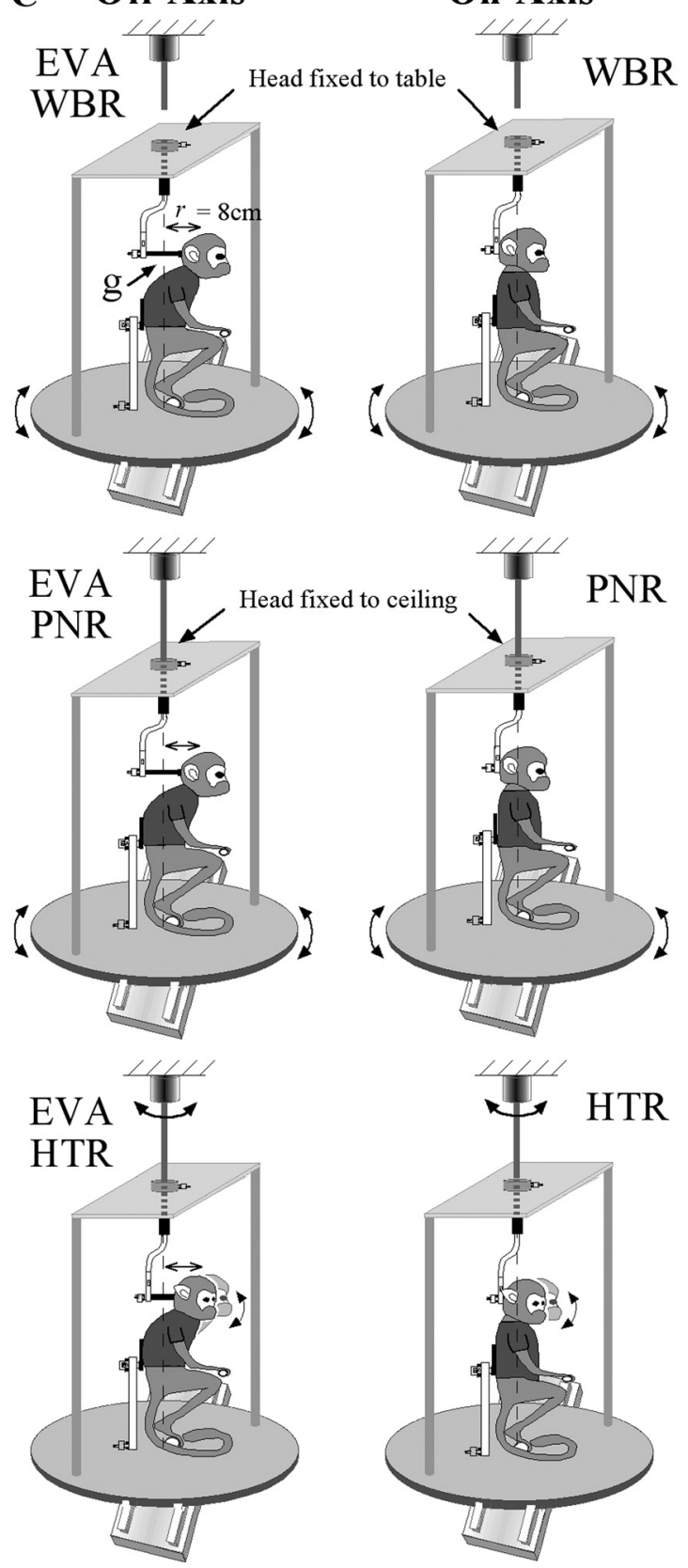

forms for sinusoidally rotating the vestibular apparatus. Measures of sled motion included linear velocity and position signals from the controller and the signal from a linear accelerometer that was mounted on the vestibular turntable. The animal was positioned on the sled so that sideto-side translations were produced. Single-unit action potentials were amplified, filtered, window-discriminated (Bak), and recorded as a digital event using a real-time clock of the acquisition system. Neural signals were also lowpass filtered (10 kHz Bessel filter) and digitized (sampling rate: $40 \mathrm{kHz}$ ) during thalamic and labyrinthine stimulation and throughout experiments. This permitted the measurement of field potentials and neural activation during stimulation and for off-line spike discrimination for data obtained during other paradigms. Eye, gaze, and head velocity signals were computed by differentiating and filtering $(20-50 \mathrm{~Hz}$ Bessel Filter) position waveforms.

Single neurons were recorded using epoxy-insulated Tungsten electrodes (4-7 M $\Omega$ impedance; FHC). Electrodes were advanced into the brain using a hydraulic microdrive (FHC) that was attached to the head implant. Electrodes were positioned within a stereotaxically placed cannula $(23 \mathrm{G})$ that guided and protected the electrode tip as it passed through the dura, cerebellar cortex, and cerebellar tentorium. The cannula defined the orientation and trajectory of the electrode. The orientation was targeted to guide the electrode through the cerebellar interpositus toward the vestibular nuclei. During the advance of each electrode, side-to-side interaural whole-body translation (Fig. 1B) was used as a search stimulus to identify regions sensitive to vestibular stimulation. This stimulus was effective for activating neurons that were located both in the vestibular nuclei and within the cerebellar interpositus. The reason for the placement of both the thalamic and labyrinthine stimulating electrodes was to experimentally distinguish IN neurons from neurons in the rFN and vestibular nuclei, which, like neurons in the IN, also demonstrate vestibular and cervical proprioceptive sensitivity. In animals in which thalamic stimulating electrodes were implanted, the ventral thalamus was electrically stimulated $(0.1 \mathrm{~ms}$ pulses, $750 \mathrm{~ms}$ interpulse interval, $50-350 \mu \mathrm{A}$ ) to identify antidromically produced field potentials in the IN. Entrance into the vestibular nuclei was coincident with the appearance of synaptically evoked field potentials following labyrinthine stimulation $(0.1 \mathrm{~ms}$ pulses, $400 \mathrm{~ms}$ interpulse interval, $50-350 \mu \mathrm{A}$ ) and the diminution of antidromic field potentials in the cerebellar nuclei evoked by electrical stimulation of the ventral thalamus. Recording locations were later confirmed based on a histological analysis.

\section{Experimental paradigms}

Several paradigms were used to compare and quantify each neuron's sensitivity to vestibular and neck proprioceptive stimulation (Fig. 1B). The following on-axis paradigms were used while the earth-vertical rotational axis of the system was aligned with the $\mathrm{C} 1-\mathrm{C} 2$ axis placing the animal in an orthograde posture:

Whole-body translation or rotation. The whole body was either translated (WBT) in a side-to-side direction or horizontally rotated (WBR) while the head was prevented from moving with respect to the trunk.

Figure 1. A, Schematic illustration of postural manipulations for the on-axis and off-axis rotation conditions. In the on-axis condition, subjects were aligned with the vertical axis of the rotational turntable and motion of the head occurred about the C1-C2 vertebral axis. In the off-axis condition, the head was displaced forward $(r=8 \mathrm{~cm})$ so that rotational movements occurred by producing lateral flexion at lower vertebral levels (C6-T3). $\boldsymbol{B}$, Schematic illustration of the on-axis experimental paradigms that were used including WBT using the sled (see arrows denoting movement), WBR using the turntable, PNR using the turntable to rotate the body while the head was restrained to the ceiling, and HTR using the ceiling motor to rotate the head while the body was held stationary in space. Shown are as follows: (a) ceiling motor, (b) fixation point to connect the earth-vertical axis of rotation to the ceiling motor or ceiling, (c) vertical rod defining earth-vertical rotational axis, (d) fixation point on the trunk, (e) turntable, ( $\mathrm{f}$ ) sled, and (g) fixation point to connect rotational axis to head. $C$, Schematic illustration of the off-axis experimental paradigms that were used including EVA-WBR ( $A$, top view illustration denotes the difference compared with WBR), EVA-PNR, and EVA-HTR. 
WBT was performed at $2 \mathrm{~Hz}, \pm 0.05-0.25 \mathrm{G}$, where $\mathrm{G}=9.8 \mathrm{~m} / \mathrm{s}^{2}$. WBR was performed at $2 \mathrm{~Hz}, \pm 10 \%$.

Passive neck rotation. The head was prevented from moving in space (using the ceiling motor) while the trunk was rotated using the vestibular turntable. PNR was performed at $2 \mathrm{~Hz} \pm 10 \%$. This paradigm is occasionally referred to as body under head in other studies (Brooks and Cullen, 2009).

Head-on-trunk rotation. The head was rotated with respect to the upper trunk using the ceiling motor. Head-on-trunk rotation (HTR) was performed at $2 \mathrm{~Hz}, \pm 10 \%$ s. Squirrel monkeys have been found to readily adapt to this paradigm without resisting the imposed movement (Gdowski et al., 2000; McCrea and Gdowski, 2003; Marlinski and McCrea, 2008a,b)

The following off-axis paradigms were used while the earth-vertical rotational axis of the system was located $8 \mathrm{~cm}$ behind the interaural plane placing the animal in a pronograde posture (Fig. 1C).

Eccentric vertical axis WBR. The head was prevented from moving with respect to the body while the whole body was horizontally rotated in space. Eccentric vertical axis (EVA)-WBR was performed at $2 \mathrm{~Hz}$, $\pm 10 \%$.

Passive eccentric vertical axis neck/upper torso rotation. The head and upper torso were prevented from moving in space (using the ceiling motor) while the lower torso and pelvic girdle was rotated using the vestibular turntable. EVA-PNR was performed at $2 \mathrm{~Hz}, \pm 10 \%$.

EVA-HTR. The head and upper torso were rotated with respect to the lower trunk using the ceiling motor. In this case, a neck proprioceptive signal was produced in addition to a linear combination of angular and linear acceleration. EVA-HTR was performed at $2 \mathrm{~Hz}, \pm 10 \%$.

After aligning the head and body with the turntable axis, most neurons were studied in response to WBR, WBT, PNR, and HTR. The posture of the animal was modified such that the head was positioned $8 \mathrm{~cm}$ in front of the axis of turntable and trunk rotation, and the responses to EVAWBR, EVA-PNR, and EVA-HTR were recorded. Since it was technically challenging to maintain neuronal isolation while modifying the animal's posture, the responses during all of the experimental paradigms were not acquired for all neurons. Descriptive statistics provide the number of neurons sampled during each paradigm.

\section{Data analysis}

Sensitivity to sinusoidal stimuli. In this study, all paradigms used sinusoidal stimuli $(2 \mathrm{~Hz})$. Response sensitivity during each paradigm was quantified using a standard technique (Gdowski et al., 2000; McCrea and Gdowski, 2003; Marlinski and McCrea, 2008a,b). Briefly, data records were edited to exclude epochs when the subject was not alert as determined by the absence of ocular saccades. The unit's instantaneous discharge rate was averaged with respect to the stimulus cycle ( $\geq 25$ cycles). Averaged responses were subjected to a least-square regressive fit with a fixed frequency sinusoidal function having a DC bias, magnitude, and phase coefficients. Gain values were computed by normalizing the magnitude coefficient by the stimulus amplitude and are reported with respect to stimulus rotational velocity $(\mathrm{sp} / \mathrm{s} / \% \mathrm{~s})$ or linear acceleration $(\mathrm{sp} /$ $\mathrm{s} / \mathrm{G})$. The corresponding response phase coefficient is reported in degrees $\left({ }^{\circ}\right)$ with respect to ipsilateral stimulus velocity or acceleration. The gain $(g)$ and phase $(\theta)$ coefficients obtained from a fit to a response to a specific paradigm are denoted with subscripts that describe the paradigm (i.e., for WBR: $g_{\text {wbr }}$ and $\theta_{\text {wbr }}$ ).

Modeling responses during EVA-WBR. When the head was eccentric from the earth-vertical axis of rotation during WBR, the vestibular end organs experienced a combination of linear translation and rotation. Responses during EVA-WBR were compared with a model that was computed based upon a linear vectorial combination of the WBR and WBT responses (WBR + WBT). The EVA-WBR response was first decomposed into rotational and translational components as follows: $H L_{v}(t)=H_{v}(t)\left[r \pi / 180^{\circ}\right]$ and $H L_{a}(t)=H_{a}(t)\left[r \pi / 180^{\circ}\right]$.

Parameters include eccentric location $(r=8 \mathrm{~cm})$, linear head velocity $\left(H L_{v}\right)$, linear head acceleration $\left(H L_{a}\right)$, angular head velocity $\left(H_{v}\right)$, and angular head acceleration $\left(H_{a}\right)$, where angular head velocity and acceleration were the first and second derivative of the stimulus waveform during EVA-WBR.

\section{Eye movement sensitivity}

Neuronal sensitivity to eye position was assessed using a multiple regression analysis of the firing rate with horizontal eye position, vertical eye position, and head position during periods of steady gaze in the absence of a visual target. The instantaneous firing rate of the neuron was binned at the data acquisition-sampling interval. Responses were averaged from data record intervals ranging in length from 10 to $100 \mathrm{~ms}$ during which no stimulus was presented. Typically, 100 averaged responses were subjected to a regressive analysis to identify relationships between firing rate and eye position. The firing rates of all neurons reported in this study lacked significant correlation with horizontal or vertical eye position.

\section{Classification of vestibular responses}

Units were categorized based upon whether they responded during WBR $\left(g_{\mathrm{wbr}}>0.1 \mathrm{sp} / \mathrm{s} / \% / \mathrm{s}\right)$ and WBT $\left(g_{\mathrm{wbt}}>100 \mathrm{sp} / \mathrm{s} / \mathrm{G}\right)$. Vestibular responses were classified according to the criteria of Duensing and Schaefer (1958) as determined by the presence of excitatory responses in phase with ipsilateral (type I) or contralateral (type II) rotational velocity during horizontal WBRs.

Each neuron was also assigned a response type based on the response to WBR and WBT. Neurons responding only during WBR were categorized as canal only. Those responding only during WBT were categorized as otolith only. Finally, those responding to both paradigms were categorized as canal and otolith. In some cases, the response to WBR was not obtained before contact with the neuron was lost. In these cases, the rotational sensitivity was estimated from the response to EVA-WBR to classify the neuron. More specifically, the response to WBT was vectorially subtracted from the response to EVA-WBR. The residual component was used as an indicator of the unit's rotational sensitivity to facilitate classification as a canal and otolith unit. The reliability of this procedure was verified from responses of neurons in which complete datasets (WBR, WBT, and EVA-WBR) were obtained. Estimates of the rotational sensitivity computed from EVA-WBR responses were compared with the rotational sensitivity determined from WBR responses. Differences between the two measures were found to be insignificant and never resulted in confounding classifications of the neuron.

It is also important to note that responses were only recorded during yaw rotations and side-to-side linear translations. The experimental setup did not permit recording responses while being translated or rotated in every direction. Some canal only and otolith only neurons could have been sensitive to linear translation or rotation in directions that were not tested. Therefore, these groups may not represent homogeneous neuron populations.

\section{Histological reconstruction}

All recording locations were determined based upon the placement of the electrode with respect to a pin that was stereotaxically positioned within the surgically implanted recording chamber. The micromanipulator and micropositioner readings were used to first map recordings with respect to the pin and were registered with atlas locations of structures. The overall location of the map was then refined based upon physiological recordings from bordering structures (e.g., the abducens nucleus, the prepositus nucleus, external cuneate nucleus, and the facial and vestibular nerves). At the completion of all experiments, one animal was perfused to reconstruct the histological location of neuronal recording sites and confirm the physiological estimates of the boundaries of the IN.

\section{Results}

\section{Electrical identification and location of single-unit recording sites}

The firing patterns of 67 neurons located in the cerebellar interpositus (IN) were recorded in three squirrel monkeys during vestibular and proprioceptive stimulation. None of these neurons demonstrated eye movement sensitivity.

IN neurons were recorded throughout the anterior IN and to a lesser extent the posterior IN. A detailed histological analysis was performed only in one animal. Figure $2 A$ details histological confirmation of recordings in that animal. Shown is a section 

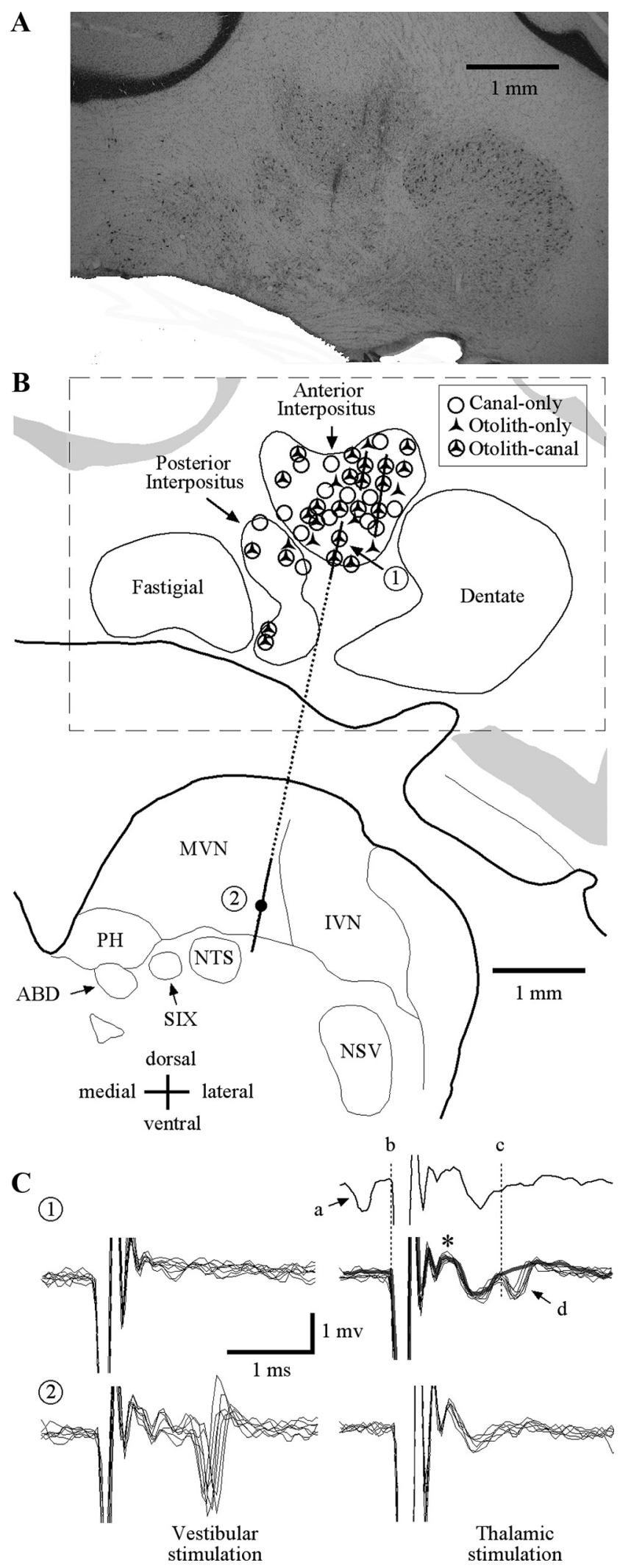

Figure 2. $A$, Histological coronal section (AP $=6.0 \mathrm{~mm}$ ) through the right cerebellum and brainstem regions including the interpositus and vestibular nuclei. Three electrode penetrations are evident from gliotic scars in the anterior interpositus. $\boldsymbol{B}$, Schematic representation of the coronal section in $\boldsymbol{A}$. Lines correspond to electrode trajectories. Symbols denote approximate locations of all neurons that were sensitive to vestibular stimulation recorded in all three animals using recording maps from each of the animals that were registered with respect to through the cerebellum $(\mathrm{AP}=6.0 \mathrm{~mm}$ ) with three electrode penetrations spanning the mediolateral extent of the anterior IN. Gliotic scars from electrode penetrations were not identified in any adjacent regions including the fastigial and dentate nuclei. The majority of the electrodes passed through the anterior IN into the lateral aspects of the medial vestibular nuclei.

Electrophysiological techniques supplemented the anatomical evidence demonstrating that the neurons recorded were in the IN. Stimulating electrodes were bilaterally implanted in the thalamus of two squirrel monkeys to identify IN neurons that projected to the thalamus. Twenty-one (of 67 tested) cerebellar IN neurons were activated from the contralateral VL. During each VL stimulus repetition, field potentials were observed (Fig. 2C, asterisk) immediately following the stimulus artifact during subthreshold stimulation $(150 \mu \mathrm{A})$. The average latency of neurons activated by VL microstimulation was $1.32 \pm 0.2 \mathrm{~ms}(n=21)$. The majority of neurons that were activated by VL stimulation $(17 / 21)$ were confirmed as antidromic activation by collisions of electrical activation with spontaneously produced action potentials (as demonstrated in the single trace in Fig. $2 C$ ). Neurons that were activated by VL stimulation but were not confirmed as antidromic activation (4/21) never produced a spontaneous action potential preceding electrical stimulation within $1.85 \mathrm{~ms}$

None of the 44 vestibular sensitive cerebellar IN neurons (of the 67 total neurons characterized) responded to ipsilateral or contralateral labyrinthine stimulation for currents up to $500 \mu \mathrm{A}$, while neurons recorded in the vestibular nuclei exhibited a distinctly different pattern of electrical activation compared with IN neurons. Units in the vestibular nuclei had activation thresholds near $100 \mu \mathrm{A}$ during labyrinthine stimulation. This combination of histologic and physiologic evidence supports that all recordings reported are from the IN.

\section{Vestibular sensitivity during WBR and WBT}

IN neurons exhibited a variety of responses to vestibular stimuli and received different combinations of rotational and translational signals arising from canals and otolith end organs. Of the 67 cerebellar IN neurons that were tested for responses to sinusoidal WBR or WBT, 44 exhibited stimulus-dependent modulations in their firing rate (Table 1). Of these, nearly half were determined to be thalamus-projecting neurons (21/44 tested).

Figure 3 illustrates the vestibular response combinations of three IN neurons that were recorded during sinusoidal WBR (2 $\mathrm{Hz}, \pm 10^{\circ}$ s, left column $)$ and WBT $(2 \mathrm{~Hz}, \pm 0.2 \mathrm{G}$, right column $)$ during which the head was restrained from moving with respect to the body. The top traces are the stimulus shown in terms of the whole-body's angular velocity and linear acceleration in space.

each other, initially based upon the stereotaxic placement of electrodes and later refined based upon physiological recording from bordering structures. C, Patterns of evoked activity for two neurons (identified as 1 and 2 in $\boldsymbol{B}$ ) recorded along a single track (dashed line) during ipsilateral vestibular labyrinth (left) and contralateral ventral thalamic (right) stimulation. The two neurons recorded along this track were located in (1) ventral IN and (2) ventral medial vestibular nuclei. The first IN unit was antidromically activated $(1 \mathrm{t}, 150 \mu \mathrm{A})$ by thalamic stimulation. The top trace shows an example of a collision where a spontaneously generated action potential (a) resulted in the absence of an electrically evoked response during thalamic stimulation (d). The same IN neuron was not activated by labyrinth stimulation $(1 \mathrm{v}, 150 \mu \mathrm{A})$. The second unit recorded in the medial vestibular nucleus, was orthodromically activated by ipsilateral vestibular labyrinth stimulation $(2 \mathrm{v}, 300 \mu \mathrm{A})$ and not by contralateral thalamic stimulation (2t, 500 $\mu \mathrm{A})$. MVN, medial vestibular nucleus; IVN, inferior vestibular nucleus; $\mathrm{PH}$, nucleus prepositus hypoglossi; NTS, nucleus of the solitary tract; ABD, abducens nucleus; SIX, nucleus salivatorius inferior; NSV, nucleus tractus spinalis; ${ }^{*}$, field potential in IN with contralateral VL stimulation. 
Table 1. Vestibular sensitivities of all neurons recorded in the cerebellar interpositus

\begin{tabular}{|c|c|c|c|c|c|c|c|}
\hline & \multicolumn{2}{|l|}{ Canal-only } & \multicolumn{2}{|c|}{ Canal and otolith } & \multicolumn{2}{|l|}{ Otolith-only } & \multirow[b]{2}{*}{ Total } \\
\hline & Type I WBR & Type II WBR & Type I WBR & Type II WBR & Ipsilateral WBT & Contralateral WBT & \\
\hline Labyrinthine activated & $0 / 3$ & $0 / 11$ & $0 / 5$ & $0 / 18$ & $0 / 2$ & $0 / 5$ & $0 / 44$ \\
\hline Total (\% activated) & $2.3 \%$ & $9.1 \%$ & $2.3 \%$ & $29.5 \%$ & $0 \%$ & $4.5 \%$ & $47.7 \%$ \\
\hline \multicolumn{8}{|l|}{$2 \mathrm{~Hz}$ WBR } \\
\hline$n$ & 3 & 11 & 5 & 18 & 2 & 5 & 44 \\
\hline$g_{\mathrm{wbr}}{ }^{a}$ & $0.28 \pm 0.07$ & $0.27 \pm 0.01$ & $0.38 \pm 0.01$ & $0.36 \pm 0.01$ & - & - & \\
\hline$n$ & 1 & 8 & 5 & 15 & 0 & 4 & 33 \\
\hline$g_{\text {eva-wbr }}{ }^{a}$ & 0.20 & $0.29 \pm 0.04$ & $0.51 \pm 0.09$ & $0.51 \pm 0.14$ & - & $0.23 \pm 0.01$ & \\
\hline$\theta_{\text {eva-wbr }}$ & 50 & $-155 \pm 8.2$ & $21 \pm 16.9$ & $-113 \pm 18$ & - & $-121 \pm 20$ & \\
\hline \multicolumn{8}{|l|}{$2 \mathrm{~Hz}$ WBT } \\
\hline$n$ & 3 & 11 & 5 & 18 & 2 & 5 & 44 \\
\hline$g_{\mathrm{wbt}}{ }^{\mathrm{c}}$ & - & - & $244 \pm 30$ & $196 \pm 4.5$ & $150 \pm 13.8$ & $138 \pm 8.9$ & \\
\hline
\end{tabular}

${ }^{a} \mathrm{sp} / \mathrm{s} /{ }^{o} / \mathrm{s}_{;}{ }^{b}$ degrees re ipsilateral stimulus velocity; ${ }^{c} \mathrm{sp} / \mathrm{s} / \mathrm{G}_{;}{ }^{d}$ degrees re ipsilateral acceleration.

Neurons were categorized as either type I or type II based on whether their response was modulated in phase with ipsilateral or contralateral velocity during WBRs (see Materials and Methods).

Most neurons responded to WBRs (37/44) like those illustrated in Figure 3. The majority of these had type II rotational responses (29/37). Most neurons also responded to side-to-side WBTs (30/44). Neurons were further characterized as canal only (Fig. 3A, top row), otolith only (Fig. $3 A$, middle row), and canal otolith (Fig. $3 A$, bottom row) based upon the presence or lack of response during WBR and WBT. Neurons responding exclusively during side-to-side WBT were the least common (otolith only, 15\%). The majority of the IN neurons either responded exclusively during rotation (canal only, 32\%) or responded during both rotation and translation (canal-otolith, 53\%). The majority of the neurons sensitive to vestibular stimulation were located throughout the anterior IN. A few neurons were also encountered in regions of the posterior interpositus. Different categories of vestibular sensitivity (i.e., canal only, etc) were found to be interspersed and evenly distributed throughout the anterior interpositus.

The response gains and phases varied among each class of neurons for both WBR and WBT (Table 1). Figure $3 B$ contains polar plots showing the distribution of the gains and phases computed from responses to $2 \mathrm{~Hz}$ WBR and WBT. Response phase is plotted with respect to ipsilateral WBR velocity and linear acceleration. The response gains and phases for canal-only and canalotolith neurons during WBR stimuli are shown in the left plot of Figure $3 B$. Type I rotational responses (filled symbols) are located in the right-hand plane and type II rotational responses (open symbols) are located in the left-hand plane. Both type II canalonly and canal-otolith neurons tended to have peak modulations that led peak contralateral whole-body velocity $\left(41 \pm 9.4^{\circ}\right.$, $\left.63 \pm 11.2^{\circ}\right)$. Fewer type I neurons were encountered; of those, the canal-only neurons tended to have peak modulations that led peak ipsilateral whole-body velocity on average by $70 \pm 17.8^{\circ}$ and were approximately in phase with rotational acceleration. Type I canal-otolith neurons also had variable response phases, but they were more closely related to ipsilateral WBR velocity (25 \pm $\left.12.4^{\circ}\right)$. The response gains and phases for otolith-only and canalotolith neurons during WBT stimuli are shown in the right side plot of Figure $3 B$. Canal-otolith neurons with type I responses during WBR are shown as filled symbols and those with type II responses during WBR are shown as open symbols. Otolith-only neurons lacked a response to WBR and are identified with trian- gle symbols. Most neurons, including both canal-otolith and otolith-only neurons, had peak modulations that lagged stimulus acceleration. This response phase is equivalent to a phase lead computed with respect to stimulus velocity. All neurons with type I WBR responses had WBT responses that lagged ipsilateral stimulus acceleration. Similarly, all neurons with type II WBR responses had WBT responses that lagged contralateral stimulus acceleration. Incongruent WBR and WBT responses (type I WBR responses with sensitivity to contralateral WBT, and vice versa) were not observed on any canal-otolith neurons.

\section{Vestibular sensitivity during eccentric WBRs}

Head movements that are produced by lateral neck flexion cause rotation and translation of the vestibular sensory epithelium in space. We hypothesized that the vestibular signals carried by IN neurons during these head movements could be estimated from responses obtained during WBR and WBT. To test this hypothesis, we quantitatively compared the responses to WBR and WBT to the responses obtained during eccentric WBRs (EVA-WBR).

The animal was placed in a pronograde posture where the rotational axis of the vestibular platform was aligned with a point of lateral neck flexion located $8 \mathrm{~cm}$ posterior to the interaural plane. In this case, the lower body and trunk were proximal to the axis of rotation and the head was located eccentric to the axis of rotation. In this posture, rotations of the whole body resulted in combined rotation and translation of the vestibular labyrinth (EVA-WBR) identical to that which would be produced if the animal had generated rotational head-on-trunk movements by lateral flexion.

Figure $4 A$ shows the responses of the three IN neurons illustrated in Figure $3 A$ during EVA-WBR. The dashed lines superimposed on the response in Figure $4 A$ are models based upon a linear combination of the vestibular sensitivities obtained from the responses to WBR and WBT. In each case, the model (WBR + WBT) closely predicted the neuron's response during combined rotation and translation (EVA-WBR) of the head in space. Responses to this combined vestibular stimulus could always be predicted using a simple additive model based upon the responses observed during WBR and WBT. We quantitatively verified this property for all neurons by comparing the gain and phase predicted by the linear model to the gain and phase of a sinusoidal fit to the response. Figure $4 B$ shows the gain and phase of the model as a function of the gain of the sinusoidal fit for 33 neurons in which the responses to all three conditions (WBR, 
WBT, and EVA-WBR) were recorded. The gain of the model was nearly identical to the gain of the sinusoidal fit for all types of IN neurons including canal-only, otolith-only, and canal-otolith neurons. A linear fit to the data in Figure $4 B$, top, showed a direct relationship between the gain of the model and fit to the data during EVA-WBR (slope \pm SD $1.04 \pm 0.02$ ). The response phase of the model was also nearly identical to the response phase from the sinusoidal fit for all types of IN neurons including canal-only, otolithonly, and canal-otolith neurons (slope \pm SD $0.96 \pm 0.02$; Fig. $4 B$, bottom). These data show that vestibular signals associated with rotation and translation carried by IN neurons are combined linearly when produced simultaneously by rotating the whole body when the head is eccentrically located within $8 \mathrm{~cm}$ of the rotational axis. This supports the hypothesis that the vestibular signals generated by producing head-on-trunk movement by lateral flexion are a linear combination of the individual rotational and translational vestibular signals carried by neurons.

This linear relationship was used to facilitate categorizing some IN neurons $(n=11)$ as canal-only, otolith-only, and canal-otolith neurons when neuronal contact was lost before the response to both WBR and WBT was obtained. In those cases, neurons were classified if their responses to EVA-WBR and at least one of the other stimuli (WBR or WBT) were obtained. The response to the missing condition (WBR or WBT) was estimated based upon a vector subtraction of the two conditions that were obtained (see Materials and Methods). This technique was performed without error for all 33 neurons in which the responses to all three stimuli were obtained.

\section{Neck proprioceptive signals during axial rotation and lateral flexion}

Proprioceptive signals have been reported on IN neurons when the head was rotated with respect to the trunk in the decerebrate cat (Boyle and Pompeiano, 1980b). We hypothesized that these signals were also present in awake monkeys and that they were produced not only during axial neck rotations but also during lateral neck flexion. The proprioceptive signals associated with the neck axial rotation were assessed by restraining the head in space while rotating the lower body using the vestibular turntable (Fig. 1B, PNR). Similarly, the proprioceptive signals associated with neck lateral flexion were assessed by rotating the lower body when the animal was in a pronograde posture in which the lower body and trunk were proximal to the axis of rotation and the head was located $8 \mathrm{~cm}$ anterior to the axis of

A

Otolith-only

B WBR
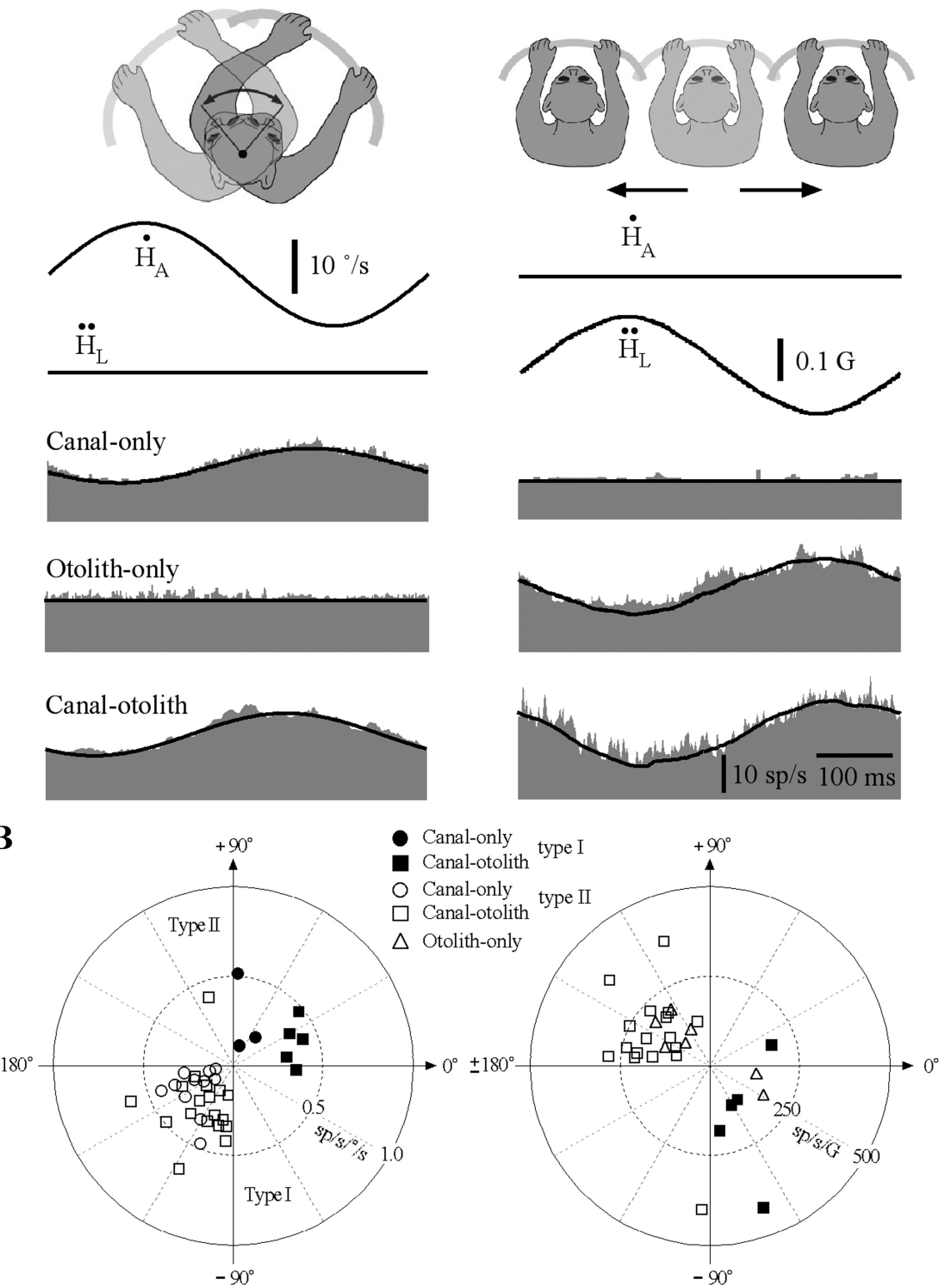

Figure 3. A, Vestibular responses of three IN neurons sensitive to sinusoidal WBR $\left(2 \mathrm{~Hz}, \pm 10^{\circ} / \mathrm{s}\right)$ or side-to-side WBT $(2 \mathrm{~Hz}, \pm$ $0.05 \mathrm{G}$ ). Each row corresponds to a different type of unit including a canal-only unit that responded only during WBR and not during side-to-side WBT, an otolith-only unit that responded during side-to-side WBT but not during WBR, and a canal-otolith unit that responded during both WBR and side-to-side WBT. Traces shown include: head velocity $(\dot{H})$, head acceleration $(\ddot{H})$, and unit discharge rate (gray fill). Schematic illustrations shown above each figure correspond to the paradigm that was used. Fits for estimating vestibular sensitivities are shown superimposed on the unit's discharge rate (see Materials and Methods). All recordings were from animal $B$. All averages included 30 stimulus cycles. All three of these example neurons also have neck sensitivity and are discussed in later figures. $\boldsymbol{B}$, Polar plots summarizing the vestibular response properties of IN neurons. Left, Gain and phase response properties of IN neurons during $2 \mathrm{~Hz}$ WBR for canal-only $(n=14)$ and canal- otolith $(n=23)$ neurons. Type I (right-hand plane, filled symbols) and type II (left-hand plane, open symbols) are shown. Gains and phases were computed with respect to rotational velocity. Right, Gain and phase response properties of IN neurons during $2 \mathrm{~Hz}$ EVA-WBR as a function of unit type (otolith-only, $n=7$; canal-otolith, $n=23$ ). Gains and phases were computed with respect to translational acceleration.

rotation (Fig. $1 B$, EVA-PNR). Figure $5 A$ illustrates the responses that were observed during $2 \mathrm{~Hz}$ PNR and EVA-PNR for the three IN neurons in Figure $3 A$ that were classified as canal-only, otolith-only, and canal-otolith neurons. The top trace in each graph is the rotational velocity of the lower body and the lower histogram represents the discharge rate of the neuron as a function of time. Only neurons that responded to vestibular stimula- 
A EVA-WBR

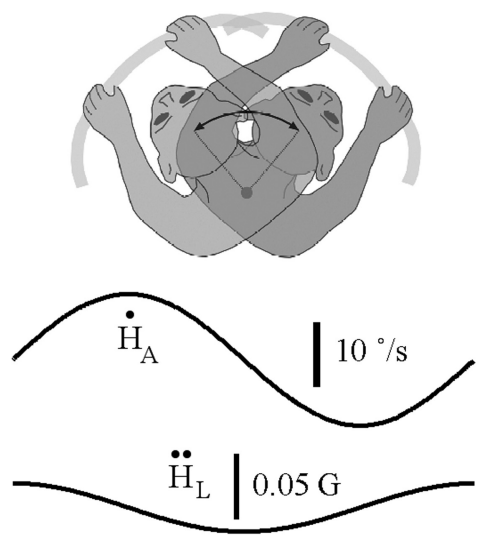

Canal-only

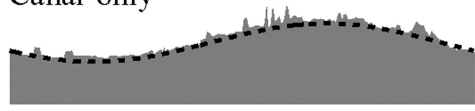

Otolith-only
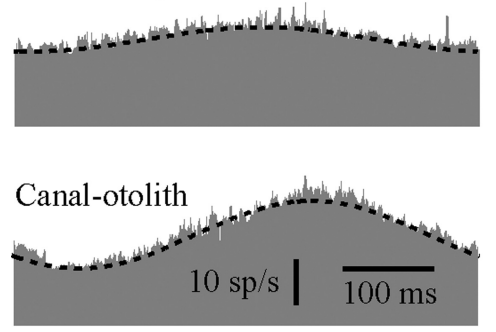
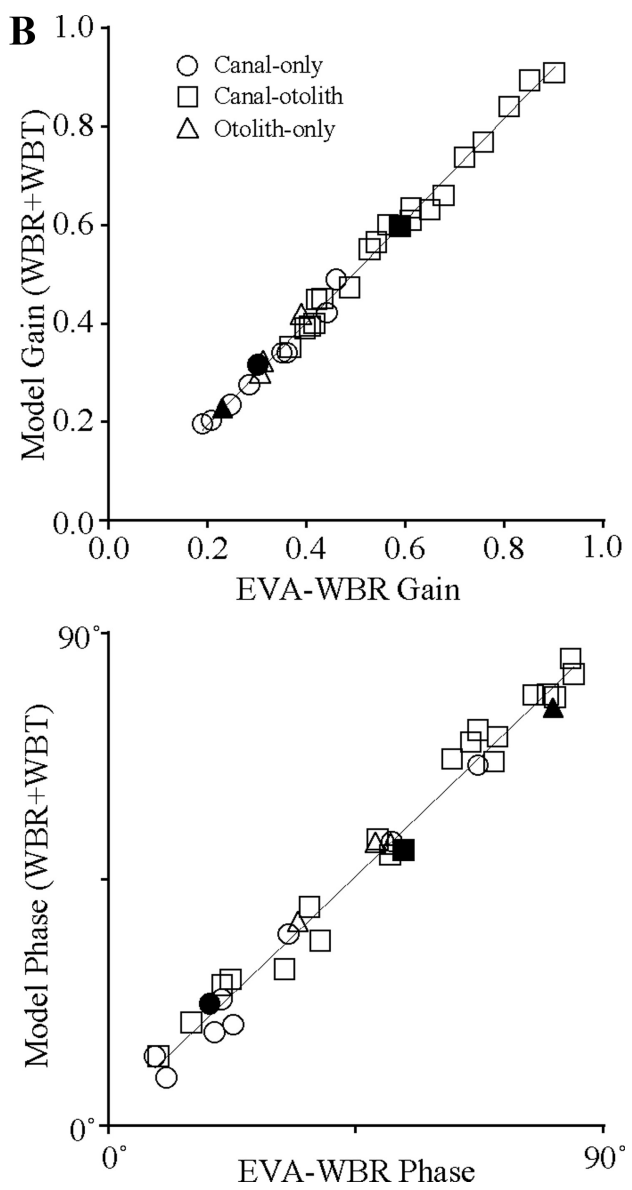

Figure 4. $\quad A$, Responses during eccentric off-axis rotations (EVA-WBR). The three neurons illustrated are the same as those in Figure 3. Responses are shown for EVA-WBR $\left(2 \mathrm{~Hz}, \pm 10^{\circ} / \mathrm{s}\right)$ while the head was located $8 \mathrm{~cm}$ in front of the earth-vertical axis. Each row corresponds to a different type of unit including a canal-only neuron, an otolith-only neuron, and a canal-otolith neuron. Traces shown include head velocity $(\dot{H})$, head acceleration $(\ddot{H})$, and unit discharge rate (gray fill). Schematic illustration at the top shows the paradigm that was used. Models superimposed on each unit's response (dashed lines) are a linear combination of the WBR and WBT response sensitivities illustrated in Figure 3 (see Materials and Methods). All recordings were from animal B. All averages included 30 stimulus cycles. $\boldsymbol{B}$, Linearity of responses during eccentric off-axis rotations (EVA-WBR). Top, Estimated gain from a linear model representing a combination of the WBR and WBT response sensitivities as a function of the gain determined by regressively fitting the neural response. The superimposed line is a linear fit (model gain $=1.04^{*} g_{\text {eva-wbr }}-0.041 \mathrm{sp} / \mathrm{s} / \% / \mathrm{s} ; R^{2}=$ 0.993). Bottom, Estimated response phase from the linear model as a function of the response phase determined by regressively fitting the neural response. The superimposed line is a linear fit (model phase $=0.96^{*} \theta_{\text {eva-wbr }}+2.9^{\circ} ; R^{2}=0.984$ ). Thirty-two neurons are shown including: 9 canal-only, 4 otolith-only, and 19 canal-otolith neurons (see legend). The filled symbols are the three neurons illustrated in Figure 3.

tion were tested for response sensitivity to neck proprioceptive stimulation. Approximately half of the cells that responded to vestibular stimulation also responded to neck proprioceptive stimulation (21/44), with most neurons active during neck rotation (18/21 units tested) and all neurons active during neck lateral flexion ( $n=21 / 21$ units tested). Table 2 summarizes the neck proprioceptive response properties for the neurons that were sensitive to either PNR or EVA-PNR.

Figure $5 B$, left column, is a polar plot that summarizes the range of gains and phases of the responses of IN neurons during passive neck rotation about the $\mathrm{C} 1-\mathrm{C} 2$ axis (PNR). Symbols denote unit category and filled and unfilled symbols denote the vestibular response type (I and II, respectively). The gains and phases of PNR responses of all neurons were located in the opposite quadrant with respect to their corresponding location in the polar plot describing vestibular sensitivities during WBR (Fig. $3 B$ ), meaning that in all cases, sensitivity to head (vestibular) rotation and neck rotation were in opposite directions.

A similar analysis was performed examining the sensitivity of neurons during lateral neck flexion (EVA-PNR) and comparing these responses to the vestibular responses of the same neurons. Figure $5 B$, right column, is a polar plot that summarizes the range of gains and phases of the responses of IN neurons during passive neck flexion about the C6-T3 axis (EVAPNR). The gains and phases of EVA-PNR responses of all neurons were also located in the opposite quadrant with respect to their corresponding location in the polar plot describing vestibular sensitivities during EVA-WBR (Fig. 3B), again demonstrating oppositely directed neck and vestibular sensitivities.

\section{Neck proprioceptive signals and their relationship to vestibular sensory signals}

A neuron's neck proprioceptive sensitivity was also contingent upon its vestibular sensitivities during WBR and WBT. Neurons sensitive to WBR (canal-only and canal-otolith neurons) were found to be sensitive to PNR, whereas neurons insensitive to WBR (otolith-only neurons) were found to be insensitive to PNR. All categories of neurons were found to be sensitive to lateral neck flexion (EVAPNR) including otolith-only neurons. This relationship suggested that the convergence of proprioceptive and vestibular sensory signals was contingent upon an individual neuron's specific vestibular signals that would be produced during different types of headon-trunk movements (axial rotation and lateral bending).

The relationship between the neck proprioceptive signals and the vestibular signals was further quantified by comparing the response gains and phases of proprioceptive and vestibular sensory signals. Figure $6 A$, top, is a plot of the gain during PNR as a function of the gain during WBR for all units tested. Two types of IN neurons were readily distinguished in Figure 6A, top, based upon the relative strength of response during PNR. Nearly one-third of the IN neurons that were sensitive to vestibular stimulation were not sensitive to rotational neck proprioceptive stimulation as defined by gain of the PNR $(<0.1 \mathrm{sp} / \mathrm{s} / \% / \mathrm{s})$. A second population of IN neurons, also sensitive to vestibular stimulation, had PNR responses. A linear fit to all neurons in Figure $6 A$, top, with significant PNR gains $(>0.1 \mathrm{sp} / \mathrm{s} / \%$ s $)$ revealed a one-to-one relationship between the gains during WBR and PNR. A similar analysis (Fig. 6A, bottom) was performed to determine whether the WBR and PNR responses were always in opposite directions. After adding $180^{\circ}$ to the PNR phase (to directly compare opposing direction) and referencing both type I and type II responses such that excitatory rotational velocity is $0^{\circ}$ phase, all head and neck sensitivity fell between the 0 and $90^{\circ}$ phase. Units that lacked responses during PNR were assigned a relative phase 
of zero. All of the neurons that exhibited similar WBR and PNR response gains also had a similar relative response phase (i.e., completely out of phase by $180^{\circ}$ ). A linear fit to all neurons in Figure $6 A$, bottom, with significant $\mathrm{PNR}$ gains $\left(>0.1 \mathrm{sp} / \mathrm{s} /{ }^{\circ} / \mathrm{s}\right)$ revealed a one-to-one relationship between the relative phase during WBR and PNR. In sum, all IN neurons that responded to PNR had WBR responses that were dynamically matched in gain and phase resulting in sensory cancelation during HTRs. This relationship was maintained regardless of the unit's vestibular response type (I or II) or classification (canal only, canal-otolith). The one exception was otolith-only neurons, which were found to be insensitive to PNR stimulation.

A gain and phase comparison identical to that performed for the WBR and PNR responses was performed for the EVAWBR and EVA-PNR responses to determine whether the vestibular and neck proprioceptive inputs were dynamically matched and in opposite directions. Figure $6 B$, top, is a plot of the gain during EVA-PNR as a function of the gain during EVA-WBR for all units tested. Again, two types of IN neurons were readily distinguished based upon the relative strength of response during EVA-PNR. One small population $(n=7)$ of IN neurons was sensitive to off-axis vestibular stimulation (EVA-WBR), and was not sensitive to lateral neck flexion (EVA-PNR). A second more predominant population $(n=21)$ of IN neurons, also sensitive to off-axis vestibular stimulation, had EVA-PNR responses with nearly identical gains. Even otolith-only neurons, which failed to respond during PNR, responded in a consistent manner during EVA-PNR. A linear fit to all neurons in Figure $6 B$, top, with significant EVA-PNR gains $\left(>0.1 \mathrm{sp} / \mathrm{s} /{ }^{\circ} / \mathrm{s}\right)$
A

PNR

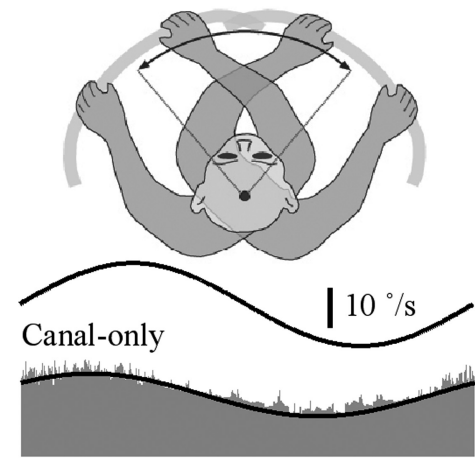

Otolith-only

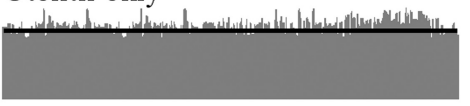

Canal-otolith
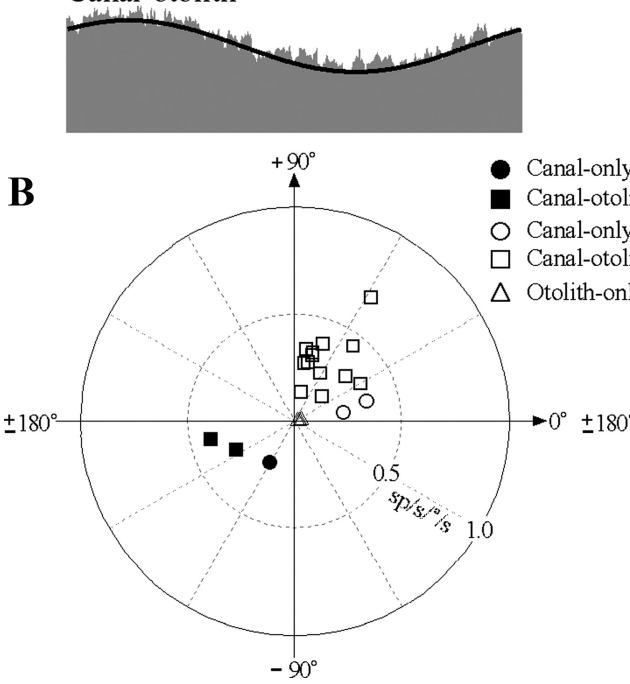

Figure 5. A, Typical responses of IN neurons during on-axis neck rotations about the C1-C2 axis (left, PNR) and off-axis neck flexion about the (6-T3 axis (right, EVA-PNR). Shown are the responses during $2 \mathrm{~Hz}$ rotations ( $\pm 10 \%$ ) for a canal-only (top), otolith-only (middle), and canal-otolith neuron (bottom). Traces shown include neck angular velocity (solid trace) and unit discharge rate (histogram). Fits for estimating the unit's gain and phase are shown superimposed on the unit's discharge rate. The neurons illustrated here are the same as in Figure 3. B, Summary of the IN neuron response properties during axial PNRs and lateral neck flexion (EVA-PNR). Left, A polar plot summarizing the gain and phase characteristics of neuron responses during PNR as a function of unit type. Right, A polar plot summarizing the gain and phase characteristics of neuron responses during EVA-PNR as a function of unit type. Filled (ipsilateral sensitive) and open (contralateral sensitive) symbols in all plots denote the unit's vestibular sensitivity.

Table 2. Neck proprioceptive sensitivities of IN neurons

\begin{tabular}{|c|c|c|c|c|c|c|}
\hline & \multicolumn{3}{|c|}{ Ipsilateral vestibular sensitivity } & \multicolumn{3}{|c|}{ Contralateral vestibular sensitivity } \\
\hline & Canal-only & Otolith-only & Canal and otolith & Canal-only & Otolith-only & Canal and otolith \\
\hline $\begin{array}{l}\text { PNR response }{ }^{a} / \text { tested } \\
2 \mathrm{~Hz} \text { PNR }\end{array}$ & $1 / 3$ & $0 / 2$ & $2 / 5$ & $2 / 11$ & $3 / 5$ & $13 / 18$ \\
\hline $\begin{array}{c}\theta_{\mathrm{pnr}}^{c} \\
2 \mathrm{~Hz} \text { WBR }\end{array}$ & -120 & - & $-161 \pm 9.8$ & $13 \pm 4$ & - & $64 \pm 17.8$ \\
\hline $\begin{array}{c}g_{\text {wbr }}{ }^{b} \\
\theta_{\text {wbr }}{ }^{c} \\
2 H z \text { EVA-PNR }\end{array}$ & $\begin{array}{l}0.20 \\
53\end{array}$ & - & $\begin{array}{r}0.33 \pm 0.05 \\
19 \pm 14.4\end{array}$ & $\begin{array}{c}0.31 \pm 0.05 \\
-165 \pm 8.8\end{array}$ & - & $\begin{array}{c}0.33 \pm 0.12 \\
-116 \pm 19\end{array}$ \\
\hline $\begin{array}{c}g_{\text {eva-pnr }}{ }^{b} \\
\theta_{\text {eva-pnr }}{ }^{c} \\
2 \mathrm{~Hz} \text { EVA-WBR }\end{array}$ & $\begin{array}{r}0.22 \\
-125\end{array}$ & - & $\begin{aligned} 0.55 & \pm 0.14 \\
-159 & \pm 8\end{aligned}$ & $\begin{array}{c}0.29 \pm 0.09 \\
11 \pm 1\end{array}$ & $\begin{array}{c}0.26 \pm 0.07 \\
53 \pm 28\end{array}$ & $\begin{array}{r}0.59 \pm 0.17 \\
64 \pm 15.5\end{array}$ \\
\hline $\begin{array}{c}g_{\text {eva-wbr }}{ }^{b} \\
\theta_{\text {eva-wbr }}{ }^{c}\end{array}$ & $\begin{array}{l}0.20 \\
50 \\
\end{array}$ & - & $\begin{array}{l}0.54 \pm 0.09 \\
20.7 \pm 16\end{array}$ & $\begin{array}{r}0.31 \pm 0.05 \\
-167 \pm 7.0 \\
\end{array}$ & $\begin{array}{r}0.28 \pm 0.05 \\
-125 \pm 24 \\
\end{array}$ & $\begin{aligned} 0.57 & \pm 0.17 \\
-115 & \pm 19\end{aligned}$ \\
\hline
\end{tabular}

${ }^{a}$ Number of units sensitive to PNR $\left(g_{\mathrm{pnr}}>0.1 \mathrm{sp} / \mathrm{s} /{ }^{\circ} / \mathrm{s}\right) ;{ }^{b} \mathrm{sp} / \mathrm{s} /{ }^{\circ} / \mathrm{s} ;$; degrees re ipsilateral stimulus velocity. 

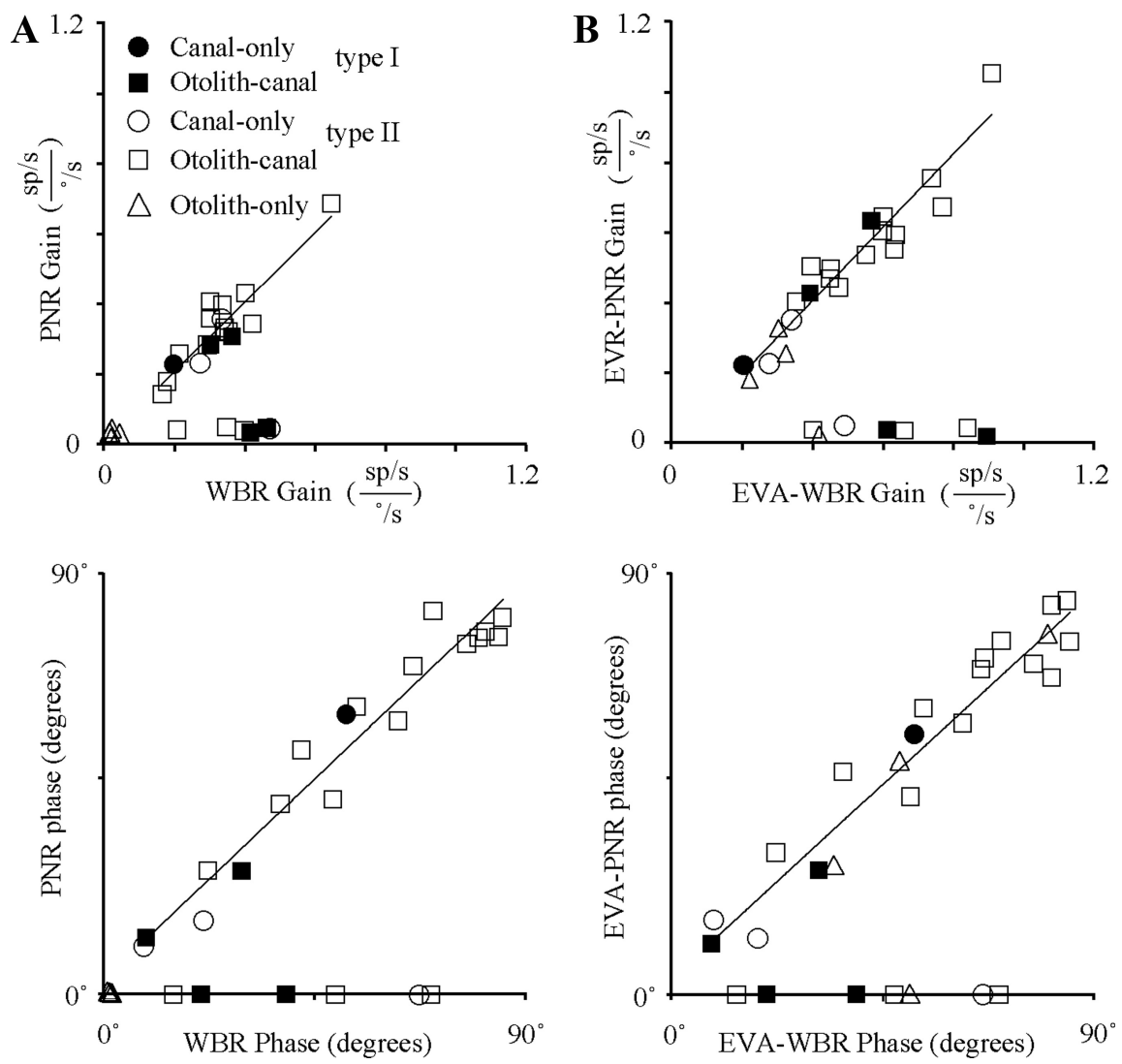

Figure 6. Comparison of neck proprioceptive and vestibular sensitivities. $A$, Top, Gain during PNR as a function of the gain during WBR. Bottom, Relative phase during PNR as a function of the relative phase during WBR. Relative phase was computed by subtracting $180^{\circ}$ from both the WBR and PNR response phase if the WBR response phase was $>180^{\circ}$. In both graphs, linear fits are shown superimposed upon the population of IN neurons having both WBR and PNR responses with significant gains $(>0.1$ $\mathrm{sp} / \mathrm{s} / \%$ s). Linear fits: Top, $g_{\mathrm{pnr}}=0.99^{*} g_{\mathrm{wbr}}+0.001, R^{2}=0.85$ and bottom, $\theta_{\mathrm{pnr}}=0.95^{*} \theta_{\mathrm{wbr}}+2.54, R^{2}=0.95$. B, Top, Gain during EVA-PNR as a function of the gain during EVA-WBR. Bottom, Relative phase during EVA-PNR as a function of the relative phase during EVA-WBR. In both right-side graphs, linear fits are shown superimposed upon the population of IN neurons having both EVA-WBR and EVA-PNR responses with significant gains $(>0.1 \mathrm{sp} / \mathrm{s} / \% \mathrm{~s})$. Linear fits: top, $g_{\text {eva-pnr }}=1.04^{*} g_{\text {eva-wbr }}+0.01$, $R^{2}=0.92$ and bottom, $\theta_{\text {eva-prr }}=0.92^{*} \theta_{\text {eva-wbr }}+3.55, R^{2}=0.93$.

revealed a one-to-one relationship between the gains during EVA-WBR and EVA-PNR. All of the neurons that exhibited similar EVA-WBR and EVA-PNR response gains also had response phases that were completely out of phase by $180^{\circ}$. A linear fit to all neurons in Figure $6 B$, bottom, with significant EVA-PNR gains $(>0.1 \mathrm{sp} / \mathrm{s} / \% / \mathrm{s})$ revealed a one-to-one relationship between the relative phase during EVA-WBR and EVA-PNR. In sum, all IN neurons that responded to PNR or EVA-PNR had vestibular responses that were dynamically matched in gain and phase. We hypothesized that the convergence of these signals would result in sensory cancelation during HTRs and lateral flexions.

\section{Vestibular sensitivity during HTR and lateral flexion}

Vestibular signals associated with the canal and otolith end organs were readily observed during movements of the whole body in space (WBR, WBT, and EVA-WBR). However, vestibular signals were absent for all neurons with neck sensitivity when the head was rotated or laterally flexed on the trunk producing the same head motion in space as the WBR and EVA-WBR stimuli.

Figure $7 A$ shows the responses of three types of IN neurons during HTRs about the C1-C2 axis including a canal-only unit, a canal-otolith unit, and an otolith-only unit. These neurons are the same as those illustrated in Figures 3-5. As noted in Figure 5, all three of these neurons have neck motion sensitivity. The top two traces in Figure $7 A$ show the vestibular stimulus in terms of the rotational velocity and linear acceleration of the head in space. Models based upon the unit's vestibular sensitivity during WBR are superimposed on the response (dashed lines) during HTRs. Both the canal-only and the canal-otolith IN neurons responded during WBR ( $g_{\mathrm{wbr}}$ $=0.32$ and $0.41 \mathrm{sp} / \mathrm{s} / \%$ s) but failed to respond similarly when the head was rotated on the trunk about the $\mathrm{C} 1-\mathrm{C} 2$ axis. The otolith-only neuron did not respond while the head was rotated on the trunk, consistent with not being sensitive to WBRs. These observations were consistent for all neurons tested. The fit to the responses (solid line) yielded nonsignificant gains during the HTR paradigm ( $g_{\text {htr }}=0.02,0.01$, and $0.02 \mathrm{sp} / \mathrm{s} /{ }^{\circ} / \mathrm{s}$ ). The average sensitivity to HTRs across the entire population for IN neurons with neck sensitivity was $0.02 \pm 0.006 \mathrm{sp} / \mathrm{s} /{ }^{\circ} / \mathrm{s}$ for canal-only $(n=3), 0.03 \pm 0.008 \mathrm{sp} / \mathrm{s} /{ }^{\circ} / \mathrm{s}$ for canal-otolith $(n=15)$, and $0.02 \pm$ $0.005 \mathrm{sp} / \mathrm{s} / \%$ for otolith-only $(n=3)$ IN neurons.

An identical analysis was performed for responses during eccentric HTRs (EVA-HTR). Figure $7 B$ shows the responses of the same three IN neurons during HTRs that involved lateral flexion of the neck (EVA-HTR). In all cases, IN neurons responded during off-axis WBR (EVA-WBR) but failed to respond similarly when the head was rotated on the trunk by neck flexion (EVA-HTR). The fit to the responses (solid line) yielded nonsignificant gains during the HTR paradigm $\left(g_{\text {eva-htr }}=0.01,0.01\right.$, and $\left.0.02 \mathrm{sp} / \mathrm{s} /{ }^{\circ} / \mathrm{s}\right)$. The average sensitivity to off-axis HTRs across the entire population for IN neurons was $0.02 \pm 0.005 \mathrm{sp} / \mathrm{s} / \%$ for canal-only $(n=3), 0.03 \pm 0.01 \mathrm{sp} / \mathrm{s} / \%$ s for canal-otolith $(n=$ $15)$, and $0.03 \pm 0.009 \mathrm{sp} / \mathrm{s} / \%$ for otolith-only $(n=3) \mathrm{IN}$ neurons.

In general, the responses during HTRs were smaller than those observed during WBR or EVA-WBR. We hypothesized that the lack of response in the majority of the neurons during HTR and EVA-HTR was the result of convergent neck proprioceptive signals that were antagonistic and specifically matched to each neuron's vestibular sensitivity to canal and otolith stimulation. To test this hypothesis we computed the ratio of the gains in the two conditions (i.e., $A_{\mathrm{htr}}=g_{\mathrm{htr}} / g_{\mathrm{wbr}}$ ), which is a quantitative measure of the relative magnitude of the HTR responses with respect to the response magnitude to WBR. These ratios were compared with the relative attenuation provided by convergent proprioceptive signals, which was quantitatively computed as the ratio of the gain during PNR $\left(g_{\text {pnr }}\right)$ and WBR $\left(g_{\text {wbr }}\right)$ (i.e., $A_{\text {pnr }}=1-g_{\text {pnr }} /$ $\left.g_{\text {wbr }}\right)$. Figure $7 C$, top, is a plot of the relative magnitude of the HTR responses $\left(A_{\mathrm{htr}}\right)$ as a function of the relative attenuation provided by proprioceptive signals $\left(A_{\mathrm{pnr}}\right)$ for all neurons in which the response to all three conditions were recorded (WBR, PNR, and HTR). In all cases, the HTR responses were either 
A

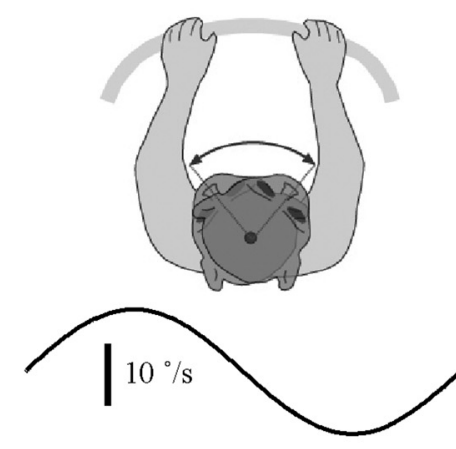

Canal-only

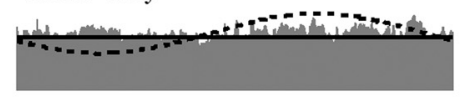

Otolith-only
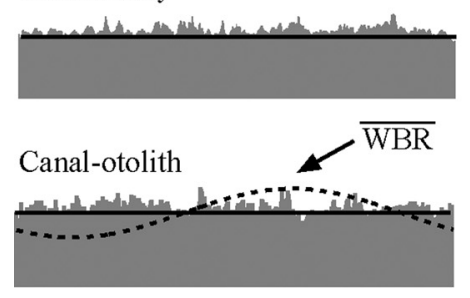

B

EVA-HTR
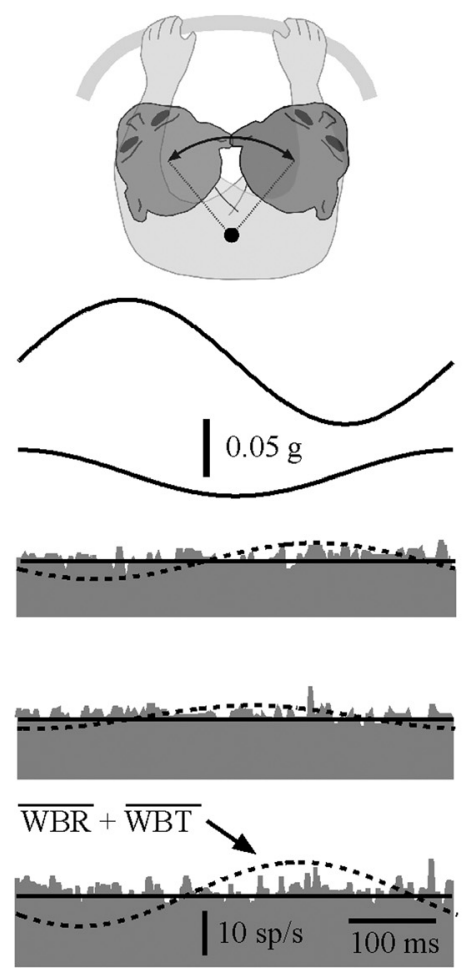
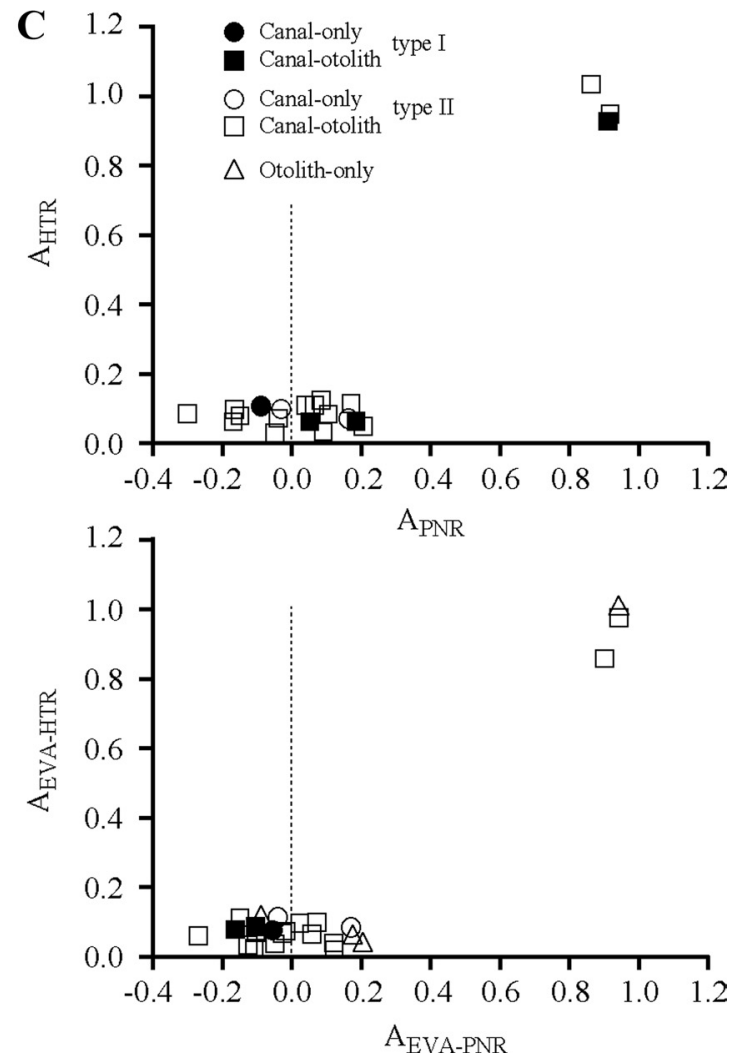

Figure 7. $\boldsymbol{A}$, Responses of three types of neurons during $\mathrm{HTRs}\left(2 \mathrm{~Hz}, \pm 10^{\circ} / \mathrm{s}\right)$ about the $\mathrm{C} 1-\mathrm{C} 2$ axis including a canal-only unit, a canal- otolith unit, and an otolith-only unit. $\boldsymbol{B}$, Responses of three types of neurons during EVA-HTR $\left(2 \mathrm{~Hz}, \pm 10^{\circ} / \mathrm{s}\right)$ that produced lateral flexion of the neck including a canal-only unit, a canal - otolith unit, and an otolith-only unit. The neurons illustrated here are the same as in Figure 3. C, Top, The relative gain during HTRs (HTR gain/WBR gain) as a function of the relative strength of on-axis rotational proprioceptive signals (1 - [PNR gain/WBR gain]). Bottom, The relative gain during eccentric HTRs (EVA-HTR gain/EVA-WBR gain) as a function of the relative strength of on-axis rotational proprioceptive signals (1 - [EVA-PNR gain/EVA-WBR gain]). Each type of neuron is identified with different symbols (see legend).

completely attenuated $\left(A_{\mathrm{htr}} \approx 0\right)$ or were similar to vestibular signals $\left(A_{\mathrm{htr}} \approx 1\right)$. All neurons that had weak responses during $\operatorname{HTR}\left(A_{\mathrm{htr}} \approx 0\right)$ had strong proprioceptive signals, which combined antagonistically with vestibular signals $\left(A_{\mathrm{pnr}} \approx 0\right)$. The three neurons that had strong responses during $\operatorname{HTR}\left(A_{\mathrm{htr}} \approx 1\right)$ had weak proprioceptive signals $\left(A_{\mathrm{pnr}} \approx 1\right)$. An identical analysis was performed for responses during EVA-WBR and EVA-PNR and is shown in Figure $7 C$, bottom. The outcome was exactly the same. In all cases, the EVA-HTR responses were either completely attenuated $\left(A_{\text {eva-htr }} \approx 0\right)$ or were similar to vestibular signals $\left(A_{\text {eva-htr }} \approx 1\right)$. These results demonstrate that the responses observed during both HTR and EVA-HTR arise as a consequence of the convergence of passive vestibular and proprioceptive sensory signals in those neurons with convergence.

\section{Discussion}

The few studies quantifying vestibular sensory signals within the IN have been conducted in decerebrate cats and even fewer studies have evaluated IN neck proprioceptive signals (Boyle and Pompeiano, 1979, 1980b; Arshavskiü Iu et al., 1980; Armstrong and Edgley, 1984). This study greatly extends our knowledge of how these two sensory signals are integrated within the IN of awake squirrel monkeys during passive head movements. IN neurons with vestibular signals exhibited two distinct types of responses when the head is rotated and translated in space with respect to the trunk. One neuron type received little or no neck proprioceptive input, whereas the second type received dynamically matched and opposing neck proprioceptive input whether tested with rotations about the $\mathrm{C} 1-\mathrm{C} 2$ axis or lateral flexion about the $\mathrm{C} 6-\mathrm{T} 3$ axis.

\section{Vestibular and neck proprioceptive signals in the} cerebellar interpositus

Some characteristics we report in IN are also observed on neurons located in the VN recorded in awake squirrel monkeys. In particular, some VN neurons show canceling vestibular and neck proprioceptive inputs for on-axis rotations similar to our WBR and PNR (Gdowski and McCrea, 2000). All of the IN cells reported here lacked eye movement-related responses, suggesting that the upstream signals from the $\mathrm{VN}$ arise from cells lacking responses correlated with eye movements; cells commonly referred to as vestibular only (Fuchs and Kimm, 1975) or noneyemovement-related (NEM) (McCrea et al., 1999). Many VN NEM neurons are sensitive to either head rotation or translation or both (Angelaki and Dickman, 2000) much like what we have reported here for IN neurons. Many, but not all, NEM VN neurons also have neck proprioceptive signals that oppose their vestibular signals during head-on-trunk movements (Boyle and Pompeiano, 1980a: Gdowski and McCrea, 2000). Horseradish peroxidase (HRP) retrograde axonal transport studies in cats describe bilateral projections from the medial and descending vestibular nuclei to the IN (Kotchabhakdi and Walberg, 1978; Oka et al., 1985), though one report in capuchin monkeys suggest these connections may be weaker in nonhuman primates (GonzaloRuiz and Leichnetz, 1990). Presumably, convergence of canal and 
otolith signals in IN could reflect convergence within neurons in the VN that project to the IN (Gdowski and McCrea, 2000).

However, the IN also receives neck proprioceptive input from the external cuneate nucleus (McCrea et al., 1977, 1978; Bakker et al., 1985), a region sensitive to complex neck rotations (Anastasopoulos et al., 1991) and the lateral reticular nucleus, where neck and utricular macular inputs combine, mostly in response cancellation (Kubin et al., 1980). So while convergence in the VN may be reflected in IN responses, it also likely that further processing involving converging sensory signals from these other nuclei contribute to vestibular-proprioceptive interactions in IN.

A most striking observation was that when a neuron exhibited neck proprioceptive sensory signals, the signals were always $\mathrm{dy}$ namically matched to the cell's vestibular sensory signals such that combined activation resulted in perfect cancelation of the two sensory signals. The elegance of this outcome is particularly striking because of the complex relationship between the vestibular end organs and cervical musculature. Unlike extraocular muscles, which align with semicircular canal planes (Graf et al., 1993), cervical spinal musculature spans multiple joints that are stretched in complex combinations with head-on-neck movements. It is hard, then, to imagine a simple mechanism by which precise dynamic mapping of vestibular and proprioceptive consequences of a head-on-neck movement occurs on a neuron-byneuron basis. The current study is limited in that we tested only two potential circumstances at one fixed radius and one frequency: yaw rotation (about the $\mathrm{C} 1-\mathrm{C} 2$ axis) and eccentric rotation (lateral flexion about the C6-T3 axis). However, such sensory cancellation at the neuronal level is likely present with other 3D head movements about multiple axes. Our study is also limited in that only passive head movements were studied; the responses of the neurons may be different with voluntary head-on-neck movements.

In a previous study in squirrel monkeys, Gdowski and McCrea (1999) reported that many VN neurons carried sensory signals describing body motion rather than head motion in space during yaw head movements about the $\mathrm{C} 1-\mathrm{C} 2$ axis. Similar physiological observations have been reported for rotational movements about the $\mathrm{C} 1-\mathrm{C} 2$ axis in the cerebellar anterior vermis (Manzoni et al., 1998, 1999) and rFN (Brooks and Cullen, 2009). Brooks and Cullen (2009) also demonstrated two populations of neurons, one population that responded to vestibular signals, but not neck signals, and a second population that responded to vestibular and neck signals perfectly out of phase and with the same gain, as in the current study. The similarity of the two studies suggests that this is a common mechanism in the cerebellar nuclei and an important feature of cerebellar function. In their study, neurons in the rFN were recorded during whole-body rotation, body-under-head rotation, and head-on-body-rotation; essentially the same as our WBR, PNR, and HTR conditions, with comparable results. Our study differs importantly in being done in a different nucleus that is involved in controlling different systems, and in extending these findings to translational stimuli and to complex neck movements. An additional, important finding in our study is that IN neurons receiving only otolith input are not sensitive to purely yaw neck rotation, but are sensitive to lateral flexion neck movement. Thus, our study is the first to demonstrate specific pairing of similar head and neck movements, which allow precise cancellation of multiple vestibular inputs by complementary multidimensional neck movements.

As the $\mathrm{rFN}$ and IN are adjacent, we considered that we might be recording fastigial neurons. However, based on stereotaxic coordinates, antidromic activation of neurons from a known IN projection area in the contralateral thalamus, and histology in one animal, this seems unlikely. Additionally, the absence of field potentials when stimulating the labyrinth eliminates the possibility that we were recording in the $\mathrm{VN}$.

\section{Targets of the cerebellar interpositus}

HRP retrograde axonal transport studies have shown that many IN cells project to the VL and the red nucleus (McCrea et al., 1978). Indeed, many of our cells were activated by electrical stimulation of VL and the response properties of those IN neurons were similar to subsets of neurons recorded in VL (Marlinski and McCrea, 2008a,b, 2009). To our knowledge, equivalent studies have never been performed in the red nucleus. Other regions receiving input from the cerebellar interpositus, such as the nucleus reticularis tegmenti pontis, pontine nuclei, inferior olive, and cerebellar cortex (McCrea et al., 1978), could also receive transformed vestibular sensory signals. Further studies are needed to determine whether these regions receive specific information describing either the body's movement in space or the head's movement in space.

\section{Functional significance}

The IN is involved in controlling upper limb movements (Thach, 1978; Stanton, 1980; Armstrong and Edgley, 1984; Hoover and Strick, 1999; Casabona et al., 2003; Monzée and Smith, 2004; Monzée et al., 2004). Early studies have shown that INs have discharge rates that are correlated with the maintenance of forearm position in space (Thach, 1978; Fortier et al., 1989, 1993). Selective inactivation of the interpositus reduces the accuracy of the distal limb during reaching tasks, suggesting a role in anticipatory, feedforward, or feedback controlled movement (Cooper et al., 2000; Martin et al., 2000; Monzée et al., 2004). Vestibular signals could be useful in maintaining the limb's position while the body is moving. Sensory signals describing movements of the body in space might be more appropriate than head-referenced vestibular signals in the control of limb movements produced with respect to the trunk. Shaikh et al. (2004) demonstrated that some rFN neurons encode translation of the body, rather than the head. Brooks and Cullen (2009) found that about one-half of neurons in the rFN demonstrated sensitivity to head and neck inputs that were of the same amplitude and opposing in phase, and speculated that these "bimodal neurons" were likely the same as those described by Shaikh et al. (2004), which coded motion in a body-centered reference frame. Although the current study did not test the reference frames for motion in IN, the similarity with the rFN bimodal neurons in that study and the body movement coding neurons in this study suggest that the IN, like the rFN, contains neurons that encode body coordinates and other neurons that encode head coordinates.

Why would a region involved primarily in controlling voluntary limb movements have constituents describing movements of either the head or body in space? One possibility, proposed by Fuller (1992), might be that different coordinates (head or body) could be useful for controlling movements in different context or different tasks. Further study of the IN during volitional limb movements with the head free to move might be the next step to understanding the consequences of matched, opposing vestibular and proprioceptive signals in this nucleus.

\section{References}

Anastasopoulos D, Mergner T, Becker W, Deecke L (1991) Sensitivity of external cuneate neurons to neck rotation in three-dimensional space. Exp Brain Res 85:565-576. Medline 
Angelaki DE, Dickman JD (2000) Spatiotemporal processing of linear acceleration: primary afferent and central vestibular neuron responses. J Neurophysiol 84:2113-2132. Medline

Armstrong DM, Edgley SA (1984) Discharges of nucleus interpositus neurones during locomotion in the cat. J Physiol 351:411-432. Medline

Arshavskiĭ Iu I, Orlovskiı̆ GN, Pavlova GA (1980) [Vestibular reactions of neurons of the cerebellar nucleus dentatus and nucleus interpositus in cats]. Neirofiziologiia 12:93-96. Medline

Bakker DA, Richmond FJ, Abrahams VC, Courville J (1985) Patterns of primary afferent termination in the external cuneate nucleus from cervical axial muscles in the cat. J Comp Neurol 241:467-479. CrossRef Medline

Belton T, McCrea RA (2000) Role of the cerebellar flocculus region in the coordination of eye and head movements during gaze pursuit. J Neurophysiol 84:1599-1613. Medline

Boyle R, Pompeiano O (1979) Sensitivity of interpositus neurons to neck afferent stimulation. Brain Res 168:180-185. CrossRef Medline

Boyle R, Pompeiano O (1980a) Responses of vestibulospinal neurons to sinusoidal rotation of neck. J Neurophysiol 44:633-649. Medline

Boyle R, Pompeiano O (1980b) Response characteristics of cerebellar interpositus and intermediate cortex neurons to sinusoidal stimulation of neck and labyrinth receptors. Neuroscience 5:357-372. CrossRef

Brooks JX, Cullen KE (2009) Multimodal integration in rostral fastigial nucleus provides an estimate of body movement. J Neurosci 29:1049910511. CrossRef Medline

Casabona A, Valle MS, Bosco G, Garifoli A, Lombardo SA, Perciavalle V (2003) Anisotropic representation of forelimb position in the cerebellar cortex and nucleus interpositus of the rat. Brain Res 972:127-136. CrossRef Medline

Chan YS, Manzoni D, Pompeiano O (1982) Response characteristics of cerebellar dentate and lateral cortex neurons to sinusoidal stimulation of neck and labyrinth receptors. Neuroscience 7:2993-3011. CrossRef

Cooper SE, Martin JH, Ghez C (2000) Effects of inactivation of the anterior interpositus nucleus on the kinematic and dynamic control of multijoint movement. J Neurophysiol 84:1988-2000. Medline

Duensing F, Schaefer KP (1958) Die Aktivität einzelner Neurone im Bereich der Vestibulariskerne bei Horizontalbeschleunigungen unter besonderer Berücksichtigung des vestibulären Nystagmus. Arch Psychiatr Nervenkr Z Gesamte Neurol Psychiatr 198:225-252. CrossRef

Eccles JC, Rantucci T, Rosén I, Scheid P, Taboriková H (1974) Somatotopic studies on cerebellar interpositus neurons. J Neurophysiol 37:1449-1459. Medline

Fortier PA, Kalaska JF, Smith AM (1989) Cerebellar neuronal activity related to whole-arm reaching movements in the monkey. J Neurophysiol 62:198-211. Medline

Fortier PA, Smith AM, Kalaska JF (1993) Comparison of cerebellar and motor cortex activity during reaching: directional tuning and response variability. J Neurophysiol 69:1136-1149. Medline

Fuchs AF, Kimm J (1975) Unit activity in the vestibular nucleus of the alert monkey during horizontal angular acceleration and eye movement. J Neurophysiol 38:1140-1161. Medline

Fuller JH (1992) Single vestibular unit recordings in the alert cat during active and passive head movements. In: The head-neck sensory motor system, Chap 44 (Berthoz A, GrafW, Vidal, PP, eds), pp 279-288. Oxford, NY: Oxford UP.

Gdowski GT, McCrea RA (1999) Integration of vestibular and head movement signals in the vestibular nuclei during whole-body rotation. J Neurophysiol 82:436-449. Medline

Gdowski GT, McCrea RA (2000) Neck proprioceptive inputs to primate vestibular nucleus neurons. Exp Brain Res 135:511-526. CrossRef Medline

Gonzalo-Ruiz A, Leichnetz GR (1990) Connections of the caudal cerebellar interpositus complex in a new world monkey (Cebus apella). Brain Res Bull 25:919-927. CrossRef Medline

Graf W, Baker J, Peterson BW (1993) Sensorimotor transformation in the cat's vestibuloocular reflex system. I. Neuronal signals coding spatial coordination of compensatory eye movements. J Neurophysiol 70:24252441. Medline

Hoover JE, Strick PL (1999) The organization of cerebellar and basal ganglia outputs to primary motor cortex as revealed by retrograde transneuronal transport of herpes simplex virus type 1. J Neurosci 19:1446-1463. Medline
Houk JC, Keifer J, Barto AG (1993) Distributed motor commands in the limb premotor network. Trends Neurosci 16:27-33. CrossRef Medline

Ito M (1984) The cerebellum and neural control. New York: Raven.

Kotchabhakdi N, Walberg F (1978) Cerebellar afferent projections from the vestibular nuclei in the cat: an experimental study with the method of retrograde axonal transport of horseradish peroxidase. Exp Brain Res 31:591-604. Medline

Kubin L, Magherini PC, Manzoni D, Pompeiano O (1980) Responses of lateral reticular neurons to sinusoidal stimulation of labyrinth receptors in decerebrate cat. J Neurophysiol 44:922-936. Medline

Magnus R (1924) Körperstellung. Springfield, VA: Amerind.

Manzoni D, Pompeiano O, Andre P (1998) Neck influences on the spatial properties of vestibulospinal reflexes in decerebrate cats: role of the cerebellar anterior vermis. J Vestib Res 8:283-297. CrossRef Medline

Manzoni D, Pompeiano O, Bruschini L, Andre P (1999) Neck input modifies the reference frame for coding labyrinthine signals in the cerebellar vermis: a cellular analysis. Neuroscience 93:1095-1107. CrossRef

Marlinski V, McCrea RA (2008a) Coding of self-motion signals in ventroposterior thalamus neurons in the alert squirrel monkey. Exp Brain Res 189:463-472. CrossRef Medline

Marlinski V, McCrea RA (2008b) Activity of ventroposterior thalamus neurons during rotation and translation in the horizontal plane in the alert squirrel monkey. J Neurophysiol 99:2533-2545. CrossRef Medline

Marlinski V, McCrea RA (2009) Self-motion signals in vestibular nuclei neurons projecting to the thalamus in the alert squirrel monkey. J Neurophysiol 101:1730-1741. CrossRef Medline

Martin JH, Cooper SE, Hacking A, Ghez C (2000) Differential effects of deep cerebellar nuclei inactivation on reaching and adaptive control. J Neurophysiol 83:1886-1899. Medline

McCrea RA, Gdowski GT (2003) Firing behaviour of squirrel monkey eye movement-related vestibular nucleus neurons during gaze saccades. J Physiol 546:207-224. CrossRef Medline

McCrea RA, Bishop GA, Kitai ST (1977) Electrophysiological and horseradish peroxidase studies of precerebellar afferents to the nucleus interpositus anterior. II. Mossy fiber system. Brain Res 122:215-228. CrossRef Medline

McCrea RA, Bishop GA, Kitai ST (1978) Morphological and electrophysiological characteristics of projection neurons in the nucleus interpositus of the cat cerebellum. J Comp Neurol 181:397-419. CrossRef Medline

McCrea RA, Gdowski GT, Boyle R, Belton T (1999) Firing behavior of vestibular neurons during active and passive head movements: vestibulospinal and other non-eye-movement related neurons. J Neurophysiol 82: 416-428. Medline

Miller LE, van Kan PL, Sinkjaer T, Andersen T, Harris GD, Houk JC (1993) Correlation of primate red nucleus discharge with muscle activity during free-form arm movements. J Physiol 469:213-243. Medline

Monzée J, Smith AM (2004) Responses of cerebellar interpositus neurons to predictable perturbations applied to an object held in a precision grip. J Neurophysiol 91:1230-1239. Medline

Monzée J, Drew T, Smith AM (2004) Effects of muscimol inactivation of the cerebellar nuclei on precision grip. J Neurophysiol 91:1240-1249. Medline

Oka H, Yoshida K, Yamamoto T, Samejima A (1985) Organization of afferent connections to the lateral and interpositus cerebellar nuclei from the brainstem relay nuclei: a horseradish peroxidase study in the cat. Neurosci Res 2:321-333. CrossRef Medline

Robinson DA (1964) The measurement of eye movement using magnetic induction in a contact lens coil. Biomed Sci Instrum 2:97-106. Medline

Shaikh AG, Meng H, Angelaki DE (2004) Multiple reference frames for motion in the primate cerebellum. J Neurosci 24:4491-4497. CrossRef Medline

Shaikh AG, Ghasia FF, Dickman JD, Angelaki DE (2005a) Properties of cerebellar fastigial neurons during translation, rotation, and eye movements. J Neurophysiol 93:853-863. Medline

Shaikh AG, Green AM, Ghasia FF, Newlands SD, Dickman JD, Angelaki DE (2005b) Sensory convergence solves a motion ambiguity problem. Curr Biol 15:1657-1662. CrossRef Medline

Stanton GB (1980) Topographical organization of ascending cerebellar projections from the dentate and interposed nuclei in Macaca mulatta: an anterograde degeneration study. J Comp Neurol 190:699-731. CrossRef Medline

Thach WT (1978) Correlation of neural discharge with pattern and force of 
muscular activity, joint position, and direction of intended next movement in motor cortex and cerebellum. J Neurophysiol 41:654-676. Medline 